\title{
Biomaterials
}

\section{Fucoidan-Functionalized Polysaccharide Submicroparticles Loaded with Alteplase for Efficient Targeted Thrombolytic Therapy \\ --Manuscript Draft--}

\begin{tabular}{|c|c|}
\hline Manuscript Number: & \\
\hline Article Type: & FLA Original Research \\
\hline Keywords: & $\begin{array}{l}\text { nanomedicine; drug delivery; targeted thrombolysis; polysaccharides; fucoidan; P- } \\
\text { selectin }\end{array}$ \\
\hline Corresponding Author: & $\begin{array}{l}\text { Cedric CHAUVIERRE, Dr } \\
\text { INSERM U1148: Laboratoire de Recherche Vasculaire Translationnelle } \\
\text { Paris, FRANCE }\end{array}$ \\
\hline First Author: & Alina Zenych, Dr \\
\hline Order of Authors: & Alina Zenych, Dr \\
\hline & Charlène Jacqmarcq \\
\hline & Rachida Aid \\
\hline & Louise Fournier \\
\hline & Laura M. Forero Ramirez, Dr \\
\hline & Thomas Bonnard, Dr \\
\hline & Denis Vivien, $\operatorname{Pr}$ \\
\hline & Didier Letourneur, Dr \\
\hline & Cedric CHAUVIERRE, Dr \\
\hline Abstract: & $\begin{array}{l}\text { Intravenous administration of fibrinolytic drugs is the standard treatment of acute } \\
\text { thrombotic diseases. However, current fibrinolytics exhibit limited clinical efficacy } \\
\text { because of their short plasma half-lives and might trigger hemorrhagic transformations. } \\
\text { Therefore, it is mandatory to develop innovative nanomedicine-based solutions for } \\
\text { more efficient and safer thrombolysis with biocompatible and biodegradable thrombus- } \\
\text { targeted nanocarrier. Herein, fucoidan-functionalized hydrogel polysaccharide } \\
\text { submicroparticles with high biocompatibility are elaborated by the inverse miniemulsion } \\
\text { / crosslinking method. They are loaded with the gold standard fibrinolytic - alteplase - } \\
\text { to direct site-specific fibrinolysis due to nanomolar interactions between fucoidan and } \\
\text { P-selectin overexpressed on activated platelets and endothelial cells in the thrombus } \\
\text { area. The thrombus targeting properties of these particles are validated in a } \\
\text { microfluidic assay containing recombinant P-selectin and activated platelets under } \\
\text { arterial and venous blood shear rates as well as in vivo. The experiments on the } \\
\text { murine model of acute thromboembolic ischemic stroke support this product's } \\
\text { therapeutic efficacy, revealing a faster recanalization rate in the middle cerebral artery } \\
\text { than with free alteplase, which reduces post-ischemic cerebral infarct lesions and } \\
\text { blood-brain barrier permeability. Altogether, this proof-of-concept study demonstrates } \\
\text { the potential of a biomaterial-based targeted nanomedicine for the precise treatment of } \\
\text { acute thrombotic events, such as ischemic stroke. }\end{array}$ \\
\hline
\end{tabular}




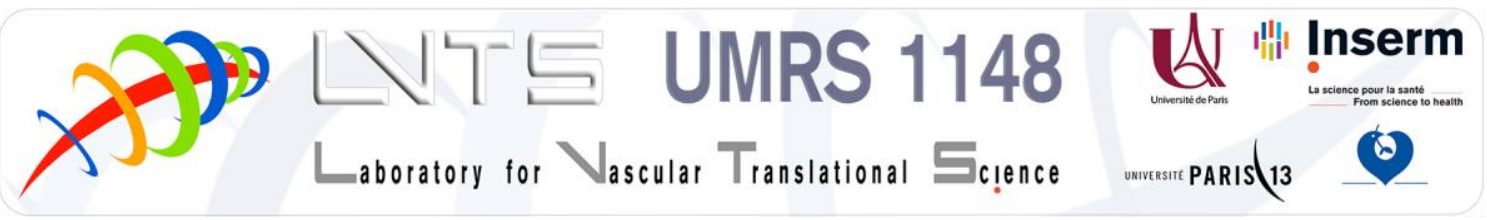

Paris, February 9, 2021

Dear Editor,

Please find enclosed the manuscript entitled "Fucoidan-functionalized polysaccharide submicroparticles loaded with alteplase for efficient targeted thrombolytic therapy" to be considered for publication in Biomaterials.

Ischemic stroke, myocardial infarction and venous thromboembolism are the main causes of death worldwide. Treatment of acute thrombotic events remains a challenge. Indeed, less than $5 \%$ of patients are injected with the standard thrombolytic drug, because of serious side effects associated with the treatment. It is thus important to create innovative nanomedicine-based solutions for more efficient and safer thrombolysis with biocompatible and biodegradable thrombus-targeted nanocarrier. In this work, polysaccharide biocompatible submicroparticles were elaborated by an original green chemistry method with all materials approved for use in humans. They were functionalized with a fucoidan that has a nanomolar affinity for the thrombus biomarker P-selectin and already used in clinical trials for molecular imaging of thrombotic diseases. Alteplase was loaded onto these hydrogel submicroparticles, the thrombolytic efficacy of the nanomedicine-based product was successfully validated in a murine model of acute ischemic stroke. This is a proof-of-concept study of the high potential of a biomaterial-based targeted nanomedicine for the precise treatment of acute thrombotic events, such as ischemic stroke.

We believe that this research article focused on the hot topics of nanomedical approaches with a translational potential for a major health issue would be of interest to the interdisciplinary audience of your highly ranked journal.

All co-authors agree with the contents of the manuscript and there is no financial interest to report. We certify that the submission is an original unpublished work and it has not been submitted to any other journal for review.

Hoping that you will find this work of interest, we are looking forward to hearing from you.

Yours sincerely,

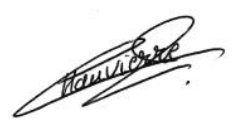

Dr. Cédric Chauvierre

Inserm, U1148, LVTS, Université Paris, CHU X. Bichat, F-75877, Paris, France

E-mail address: cedric.chauvierre@inserm.fr

Tel: (33) 14025 8600; Fax: (33) 140258602 
Fucoidan-Functionalized Polysaccharide Submicroparticles Loaded with Alteplase for Efficient Targeted Thrombolytic Therapy

Alina Zenych ${ }^{1}$, Charlène Jacqmarcq ${ }^{2, \#}$, Rachida Aid $^{1,3, \#}$, Louise Fournier ${ }^{1}$, Laura M. Forero Ramirez $^{1}$, Thomas Bonnard ${ }^{2}$, Denis Vivien ${ }^{2,4, \#}$, Didier Letourneur ${ }^{1, \#}$, and Cédric Chauvierre ${ }^{1, *}$

${ }^{1}$ Université de Paris, Université Sorbonne Paris Nord, UMR S1148, INSERM, F-75018 Paris, France

${ }^{2}$ INSERM U1237 Physiopathology and Imaging of Neurological Disorders (PhIND), Institut Blood and Brain@ Caen Normandie (BB@C), GIP Cyceron, 14074, Caen, France

${ }^{3}$ Université de Paris, FRIM, UMS 034, INSERM, F-75018 Paris, France

${ }^{4}$ Department of Clinical Research, Caen Normandie University Hospital (CHU), 14074 Caen, France.

* Corresponding author: Université de Paris, Université Sorbonne Paris Nord, UMR S1148, INSERM, F-75018 Paris, France

E-mail address: cedric.chauvierre@inserm.fr

\# Equal contribution 


\title{
Fucoidan-Functionalized Polysaccharide Submicroparticles Loaded with Alteplase for
} Efficient Targeted Thrombolytic Therapy

\author{
Alina Zenych ${ }^{1}$, Charlène Jacqmarcq, ${ }^{2, \#}$, Rachida Aid ${ }^{1,3, \#, ~ L o u i s e ~ F o u r n i e r ~}{ }^{1}$, Laura M. Forero \\ Ramirez $^{1}$, Thomas Bonnard ${ }^{2}$, Denis Vivien ${ }^{2,4, \#}$, Didier Letourneur ${ }^{1, \#}$, and Cédric Chauvierre ${ }^{1, *}$ \\ ${ }^{1}$ Université de Paris, Université Sorbonne Paris Nord, UMR S1148, INSERM, F-75018 Paris, \\ France \\ ${ }^{2}$ INSERM U1237 Physiopathology and Imaging of Neurological Disorders (PhIND), Institut \\ Blood and Brain@ Caen Normandie (BB@C), GIP Cyceron, 14074, Caen, France \\ ${ }^{3}$ Université de Paris, FRIM, UMS 034, INSERM, F-75018 Paris, France \\ ${ }^{4}$ Department of Clinical Research, Caen Normandie University Hospital (CHU), 14074 Caen, \\ France.
}

* Corresponding author: Université de Paris, Université Sorbonne Paris Nord, UMR S1148, INSERM, F-75018 Paris, France

E-mail address: cedric.chauvierre@inserm.fr

\# Equal contribution

\begin{abstract}
Intravenous administration of fibrinolytic drugs is the standard treatment of acute thrombotic diseases. However, current fibrinolytics exhibit limited clinical efficacy because of their short plasma half-lives and might trigger hemorrhagic transformations. Therefore, it is mandatory to develop innovative nanomedicine-based solutions for more efficient and safer thrombolysis with biocompatible and biodegradable thrombus-targeted nanocarrier. Herein, fucoidanfunctionalized hydrogel polysaccharide submicroparticles with high biocompatibility are elaborated by the inverse miniemulsion / crosslinking method. They are loaded with the gold standard fibrinolytic - alteplase - to direct site-specific fibrinolysis due to nanomolar interactions between fucoidan and P-selectin overexpressed on activated platelets and endothelial cells in the thrombus area. The thrombus targeting properties of these particles are validated in a microfluidic assay containing recombinant $\mathrm{P}$-selectin and activated platelets under arterial and venous blood shear rates as well as in vivo. The experiments on the murine model of acute thromboembolic ischemic stroke support this product's therapeutic efficacy, revealing a faster recanalization rate in the middle cerebral artery than with free alteplase, which reduces post-ischemic cerebral infarct lesions and blood-brain barrier permeability. Altogether, this proof-of-concept study demonstrates the potential of a biomaterial-based targeted nanomedicine for the precise treatment of acute thrombotic events, such as ischemic stroke.
\end{abstract}

Keywords: nanomedicine, drug delivery, targeted thrombolysis, polysaccharides, fucoidan, Pselectin 


\section{INTRODUCTION}

Acute thrombotic pathologies such as myocardial infarction, ischemic stroke, and venous thromboembolism remain a major global healthcare challenge contributing to a significant number of deaths and disabilities.[1] Current thrombolytic therapy, the intravenous injection of Plasminogen Activators (PA), is administrated to lyse a clot-induced vascular occlusion and restore the blood flow in the vessel. The recombinant tissue plasminogen activator (rtPA) is the most commonly applied clot-busting drug in clinics and the only one approved to treat acute ischemic stroke.[2] rtPA is a fibrin-specific serine protease that activates the endogenous proenzyme plasminogen and converts it to the active form plasmin, thus, degrading the thrombus fibrin network. However, systemic delivery of rtPA is limited by the rapid drug elimination (half-life 4-6 min), physiological deactivation by its antidotes such as Plasminogen Activator Inhibitors (PAI-1 and PAI-2), and deleterious side-effects such as intracranial hemorrhages.[3] This restricts its use to a narrow therapeutic window (4.5 h of stroke symptom onset when injected alone and $6 \mathrm{~h}$ when combined with mechanical thrombectomy) beyond which the deleterious effects of rtPA overcome its benefits. Moreover, the rate of acute recanalization after intravenous administration of rtPA is low: only $\sim 30 \%$ of patients experienced full or partial recanalization.[4]

Therapeutic strategies that intend to address the challenges of thrombolytic therapy and boost survival rates remain of great clinical interest. Certainly, novel thrombolytic molecules are being researched to increase reperfusion, improve safety, and protect the brain neurovascular unit.[5,6] Apart from that, nanomedical approaches for the targeted delivery of thrombolytic agents have been intensively proposed.[7] Korin et al. reported the microaggregates of poly (lactic-co-glycolic acid) (PLGA) nanoparticles (NPs) dissociated into rtPA-bearing nanocompounds when exposed to abnormally high hemodynamic shear stress, typical for the vascular occlusions, that performed effective thrombolysis in several preclinical 
models.[8] Colasuonno et al. formulated rtPA-loaded discoidal porous nanoconstructs from a mixture of PLGA and polyethylene glycol (PEG) with high thrombolytic potential presumably attributed to the erythrocyte-mimicking shape of the NPs and their deformability, leading to efficient circulation profiles and accumulation on the clot.[9] While these nanosystems with passive targeting succeeded in a promising thrombolytic efficacy in preclinical studies, more recent and advanced examples are formulated with actively targeted nanocarriers.

Active targeting permits drug accumulation specifically at the thrombus site and has the potential to enhance the enzyme penetration into deeply localized thrombi. Apart from the magnetic nanoparticle targeting under an external magnetic field, active blood clot targeting is currently achieved by directing the functionalized NPs towards fibrin or activated platelets (mostly integrin GPIIb/IIIa and less adhesion receptor P-selectin) with antibodies and/or peptides. Notably, a theranostic system for thrombus molecular imaging and targeted therapy was developed by Zhou et al. when rtPA was encapsulated into the $\mathrm{Fe}_{3} \mathrm{O}_{4}$-based PLGA NPs, and a cyclic arginine-glycine-aspartic acid (cRGD) peptide was grafted onto the chitosan surface to target GPIIb/IIIa on activated platelets.[10] Nevertheless, both antibodies and peptides have their limitations for targeted drug delivery. The immunogenicity, purity, and sufficient circulation time are the main concerns of the application of the antibodies[11], while peptides might suffer from weak binding affinity, immunogenicity, a high costs of peptide synthesis, and metabolic instability with fast renal clearance due to their small sizes.[12]

An effective alternative could be the nanoparticle functionalization with fucoidan,[13] a naturally-occurring algae-derived sulfated polysaccharide that exhibits a strong and specific tropism for the P-selectin overexpression in cardiovascular pathologies.[14,15] Fucoidan emerged as an affordable, high-quality targeting ligand to P-selectin that was prior validated by our group on various polysaccharide-based nano- \& microsystems for molecular diagnostics and targeted therapy.[16-20] Following the obtention of the label "raw materials for 
pharmaceutical uses" in 2015 in France, it became a part of the large-scale European Union project NanoAthero as a contrast agent for Single-Photon Emission Computed Tomography (SPECT) imaging in human atherothrombosis, coordinated by our laboratory.[21] The first in the world Phase I clinical trial on intravenous delivery of fucoidan radiolabeled by Technetium99m reported its safety and favorable biodistribution,[22] while Phase IIa for the imaging of deep vein thrombosis and pulmonary embolism is ongoing.

It is critical to ensure an excellent safety profile of the designed nanocarrier for targeted thrombolysis in future clinical translation by selecting biocompatible and fully biodegradable materials with the U.S. Food and Drug Administration (FDA)-approval.[23] Contrary to the attractiveness of synthetic polymers such as PLGA, the NPs made of polysaccharides are explored to a lesser degree for thrombolytic therapy. Yet, they benefit from the general advantages of natural polymers: biocompatibility, low cost, and hydrophilicity. Polysaccharide hydrogels, which are crosslinked three-dimensional polymer networks, absorb large quantities of water and can effectively load macromolecules with high encapsulation efficiency,[24] including plasminogen activators. Few publications reported the nanoformulations with chitosan, a cationic chitin-derived polysaccharide that can form polyelectrolyte complexes with negatively charged molecules.[25] For instance, superior thrombolytic potential in vivo was demonstrated on self-assembled chitosan NPs crosslinked with sodium tripolyphosphate and loaded with urokinase.[26] Liao et al. formulated the lumbrokinase-bearing NPs from quaternized derivative of chitosan - N,N,N-Trimethyl Chitosan covalently grafted with cRGD peptide to target GPIIb/IIIa receptors that could accelerate thrombolysis.[27]

Dextran, an exocellular bacterial water-soluble polysaccharide, is extensively employed in clinics, particularly in its low molecular weight (40 and $70 \mathrm{kDa}$ ), for plasma volume expansion, thrombosis prophylaxis, peripheral blood flow enhancement, artificial tears, etc. [28] Dextran coating of magnetic NPs is applied to ensure their environmental stability and prolong 
the blood circulation time.[29,30] However, there is no reported exclusively dextran

nanocarrier with hydrogel structure for thrombolytic application to our knowledge. Meeting the requirements of biocompatibility, biodegradability, non-immunogenicity, dextran stands out as an attractive polymer to design an alteplase delivery system.

Herein, we fabricated novel fucoidan-functionalized dextran submicroparticles (SPs) by a green chemistry method using fully biodegradable and biocompatible compounds, all of them approved by the FDA. After physico-chemical and biosafety characterization of these hydrogellike SPs, rtPA was loaded onto the SPs with a high encapsulation capacity, and its release in saline and in vitro amidolytic and fibrinolytic activities were tested. Through the in vitro microfluidic experiments under continuous arterial or venous flow, we provided evidence that fucoidan-functionalized SPs (Fuco-SPs) have a high and specific affinity to P-selectin and accumulate on activated platelet aggregates. Also, these particles bind to the thrombi in vivo. Finally, rtPA-associated Fuco-SPs proved superior in vivo thrombolytic efficacy in a mouse stroke thrombin model with a faster vessel recanalization that minimized cerebral tissue damage: the post-ischemic lesion and blood-brain barrier (BBB) permeability.

\section{MATERIALS AND METHODS}

Materials: Dextran $40 \mathrm{kDa}$ and TRITC-dextran $40 \mathrm{kDa}$ were provided by TdB Consultancy (Uppsala, Sweden). Fucoidan $(\mathrm{Mn}=18 \mathrm{kDa} / \mathrm{Mw}=104 \mathrm{kDa})$ was a gift from Algues \& Mer (Ouessant, France). Sodium trimetaphosphate (STMP), methylene blue hydrate, and Human Serum Albumin (HSA) were purchased from Sigma-Aldrich (Saint-QuentinFallavier, France). Polyglycerol polyricinoleate (PGPR) was obtained from Palsgaard France S.A.S. (Lyon, France). Vegetable (sunflower) oil was purchased from a local supermarket. The

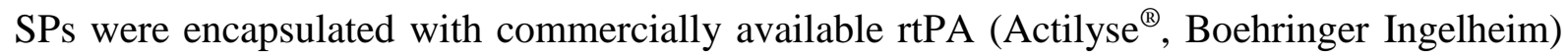
that was reconstituted at $1 \mathrm{mg} / \mathrm{ml}$, aliquoted, and stored at $-80^{\circ} \mathrm{C}$. Chromatography paper was 
obtained from GE Healthcare (Chicago, Illinois, United States). Fibrillar type I collagen Horm ${ }^{\circledR}$ was obtained from Takeda (Linz, Austria). 96-Well Cell Culture Plates (Costar) were obtained from Corning Incorporated. PPACK (Phe-Pro-Arg-Chloromethylketone) $75 \mu \mathrm{M}$ tubes were purchased from Cryopep (Montpellier, France). Flow chambers (Vena8 Fluoro+) were provided from Cellix Ltd (Dublin, Ireland).

Submicroparticle synthesis: Polysaccharide submicroparticles (SPs) were obtained via a water-in-oil (w/o) emulsification combined with a crosslinking process.[16,17] Polysaccharide solution (300 mg/ml, $6 \mathrm{M} \mathrm{NaCl}$ ) was prepared as a mixture of dextran 40 and 5\% TRITC-dextran 40 (for fluorescent SPs). To synthesize functionalized SPs with fucoidan (Fuco-SPs), 10\% w/w of fucoidan was added. Table S1, Supplementary Data describes the synthesis parameters of SPs.

First, the organic phase of $15 \mathrm{~mL}$ of sunflower oil and 6\% w/v PGPR in Falcon ${ }^{\circledR} 50 \mathrm{~mL}$ was prepared and cooled down for $20 \mathrm{~min}$ at $-20^{\circ} \mathrm{C}$. In the meantime, 1,200 $\mathrm{mg}$ of the polysaccharide solution was incubated with $120 \mu \mathrm{L}$ of $10 \mathrm{M} \mathrm{NaOH}$ under magnetic stirring for $10 \mathrm{~min} .240 \mu \mathrm{L}$ of STMP solution ( $30 \% \mathrm{w} / \mathrm{v}$ in water) was added into the aqueous phase under magnetic stirring and mixed for 20 seconds on ice. Next, emulsification was achieved by the dropwise injection of $600 \mu \mathrm{L}$ of the aqueous phase into the organic phase and dispersed with a stand-disperser (Polytron PT 3100, dispersing aggregate PT-DA 07/2EC-B101, Kinematica, Luzernerstrasse, Switzerland) at 30,000 rpm for $4 \mathrm{~min}$ on ice. The obtained w/o emulsion was transferred into $50^{\circ} \mathrm{C}$ for the crosslinking reaction of polysaccharides with STMP for $20 \mathrm{~min}$. The crosslinked suspension was washed in $30 \mathrm{~mL}$ PBS 10x for 40 min under high magnetic stirring at $750 \mathrm{rpm}$. The mixture was then centrifuged (BR4i, JOUAN SA, Saint Herblain, France) for $10 \mathrm{~min}$ at $3,000 \mathrm{~g}$ in Falcon tubes. The organic phase was recovered and ultracentrifuged (Optima MAX-XP, Ultracentrifuge, Beckman Coulter, Brea, California, United States) in PBS for $45 \mathrm{~min}$ at $15,000 \mathrm{~g}$. The obtained pellet was washed by 
ultracentrifugation 2 times in $0.04 \%$ Sodium Dodecyl Sulfate (SDS) solution and then 3 times in ultrapure water to purify the SPs. The resulting SPs were suspended in water or $0.9 \% \mathrm{NaCl}$ with $0.02 \%$ Tween 20 (Sigma) and stored at $4^{\circ} \mathrm{C}$.

Cell culture and cytotoxicity assay: To evaluate the cytotoxicity of the SPs, Fluorometric Cell Viability Assay (Resazurin) was used on confluent Human Umbilical Vein Endothelial Cells (HUVECs). The cells were cultured in DMEM supplemented with 10\% (v/v) fetal bovine serum, $4 \mathrm{mmol}$ of l-glutamine, 100 units $/ \mathrm{ml}$ of penicillin, and $100 \mu \mathrm{g} / \mathrm{ml}$ of streptomycin and kept in an incubator at $37{ }^{\circ} \mathrm{C}$ in a humidified atmosphere of $5 \% \mathrm{CO}_{2}$. Cells were seeded into 96-well plates to adhere, 10,000 cells per well. Following $24 \mathrm{~h}$ of incubation to reach $\sim 80 \%$ confluency, the medium in the wells was changed to the one containing the SPs at concentrations ranging from 0.1 to $1.5 \mathrm{mg} / \mathrm{ml}$ and cultured for another $24 \mathrm{~h}$. The SPs were prior sterilized under the UV light for $15 \mathrm{~min}$. Next, the medium was replaced with $100 \mu \mathrm{L} 10 \%$ Resazurin solution, and the plates were covered in foil and incubated for $2 \mathrm{~h}$. Culture media were used as a positive control. The Resazurin's fluorescent signals were monitored using 540 $\mathrm{nm}$ excitation and $590 \mathrm{~nm}$ emission wavelengths on Infinite ${ }^{\circledR} 200 \mathrm{PRO}$ microplate reader (TECAN Group Ltd., Mannedorf, Switzerland). The obtained fluorescence (Fl) values were blank corrected, and the relative cell viability was expressed as $\mathrm{Fl}_{\mathrm{SPs}} / \mathrm{Fl}_{\text {control }} \times 100 \%$, where $\mathrm{Fl}_{\text {control }}$ was obtained in the absence of the SPs. The experiment was performed in hexaplicate.

To examine the potential cell cytoskeleton organization mediated by Fuco-SPs, HUVECs cells were cultured in 8-well Lab-Tek II Chamber Slide w/Cover (Lab-Tek ${ }^{\circledR}$, Thermo Fischer Scientific, Massachusetts, United States) with 10,000 cells per well. The wells' medium was changed $24 \mathrm{~h}$ after to the one containing TRITC-Fuco-SPs at $1.5 \mathrm{mg} / \mathrm{ml}$ and was incubated for another $24 \mathrm{~h}$. Cells cultured in the medium without the SPs were set as control. Next, cells were fixed with $4 \%$ paraformaldehyde for $30 \mathrm{~min}$ at room temperature (RT). After rinsing with PBS, cells were labeled and permeabilized with the $200 \mu 1$ mixture of FITC-Phalloidin (1:200, 
Sigma-Aldrich, USA) / DAPI (1:100, Thermo Fisher Scientific, Massachusetts, United States)

/ 0.01\% v/v Tween 20 in PBS and incubated under low agitation for $1 \mathrm{~h}$ at RT. The cells were afterward washed 3 times with PBS. The support of the chamber slides was removed, and the slides were mounted with a few drops of the aqueous mounting medium and kept at $4{ }^{\circ} \mathrm{C}$ until visualization with the confocal microscope (Zeiss LSM 780, Iena, Germany).

Hemocompatibility test: Hemolysis assay was adapted from the publication[17] and performed on washed isolated murine erythrocytes. Murine blood was collected in sodium citrate $3.8 \%(\mathrm{w} / \mathrm{v})$ and centrifuged at $800 \mathrm{~g}$ for $5 \mathrm{~min}$ to isolate red blood cells. The supernatant was removed, and the pellet of erythrocytes was resuspended at $20 \%(\mathrm{v} / \mathrm{v})$ in distilled water (positive control, 100\% hemolysis), normal saline (negative control, no hemolysis), and the Fuco-SPs at concentrations from 0.1 to $1.5 \mathrm{mg} / \mathrm{ml}$ in Eppendorf. The tubes were incubated on a rotator at $37{ }^{\circ} \mathrm{C}$ for $1.5 \mathrm{~h}$ and then centrifuged at $3,000 \mathrm{~g}$ for $5 \mathrm{~min}$. The absorbance (A) of the supernatants was measured on Infinite ${ }^{\circledR} 200$ PRO microplate reader (TECAN Group Ltd., Mannedorf, Switzerland) at $590 \mathrm{~nm}$. Each sample was run in triplicate. The percentage of hemolysis was determined by the formula: Hemolysis degree $(\%)=100 \% \mathrm{x}\left(\mathrm{A}_{\text {sample }}-\mathrm{A}_{\text {negative }}\right.$ control $) /\left(\mathrm{A}_{\text {positive control - }} \mathrm{A}_{\text {negative control }}\right)$.

Physico-chemical characterization: The submicroparticle (SP) formulations were studied for particle morphology, size and zeta potential distributions, mass concentration, and elemental composition.

Particle morphology was visualized by Transmission Electron Microscopy (TEM) (Philips FEI Tecnai 12, Amsterdam, Netherlands), negatively stained with 1\% (w/v) uranyl acetate for 5 minutes, and Environmental Scanning Electron Microscopy (ESEM) (Philips XL30 ESEM-FEG, Amsterdam, Netherlands). Hydrodynamic size and Zeta potential ( $\zeta$ potential) were measured by Dynamic Light Scattering (DLS) and Electrophoretic Light Scattering (ELS), respectively (Zetasizer Nano ZS, Malvern Instruments SARL, Orsay, 
France). Samples were diluted in distilled water or saline for size and in $1 \mathrm{mM} \mathrm{KCl}$ for $\zeta$ potential determination. All runs were performed at $25^{\circ} \mathrm{C}$ in triplicate.

Mass concentration was determined by freeze-drying. An elemental analyzer-mass spectrophotometer was used for the quantification of the sulfur (presence of fucoidan). To prove the crosslinking with STMP, the total reflection X-ray fluorescence spectroscopy (TXRF) technique was applied to quantify the phosphorus content on the SPs (S2 PICOFOX Bruker, Massachusetts, United States).

Sulfate and fucoidan quantification: The sulfate content of fucoidan was determined by a semi-quantitative solid-phase colorimetric assay.[72] Briefly, $5 \mu \mathrm{L}$ of Fuco-SPs in suspension at a concentration of $2 \mathrm{mg} / \mathrm{ml}$ were dropped on a piece of Whatman Chromatography paper grade 1 . This was repeated 5 times on the same point, allowing the paper to dry at $50^{\circ} \mathrm{C}$ in between. The paper was first soaked into a methanol/acetone (6:4) solution for $3 \mathrm{~min}$ and then into a methanol/acetone/water (6:4:15) solution with $50 \mathrm{mM} \mathrm{HCl}$ and $0.1 \%$ w/w methylene blue for 10 min. Finally, the paper was extensively washed with acetic acid/methanol/acetone/water (5:6:4:75) until no coloration was detected in the washing solution. The paper was then transferred to the Eppendorf, containing $0.5 \mathrm{~mL}$ methanol with $2 \% \mathrm{w} / \mathrm{v}$ SDS, and incubated for $15 \mathrm{~min}$ at $50^{\circ}$ C. $0.2 \mathrm{~mL}$ of the extracted dye was placed in a 96-well plate, and its concentration was determined by reading absorbance at $663 \mathrm{~nm}$ on an Infinite ${ }^{\circledR}$ 200 PRO microplate reader (TECAN Group Ltd., Mannedorf, Switzerland). Standard curves were obtained from fucoidan in solution with known concentrations.

Loading rtPA on the SPs: rtPA was immobilized onto the SPs by adsorption. $100 \mu 1$ of SPs $(5 \mathrm{mg} / \mathrm{ml})$ was mixed with $100 \mu \mathrm{l}$ of $\mathrm{rtPA}(1 \mathrm{mg} / \mathrm{ml})$ in ultrapure water and then incubated for $1 \mathrm{~h}$ at RT. Free unabsorbed rtPA was removed by 3 cycles of ultracentrifugation (15 min, $15,000 \mathrm{~g}$ ). The SPs with adsorbed rtPA (rtPA-SPs) were resuspended in water and used for the drug loading efficiency quantification. 
Drug encapsulation efficiency: The amount of rtPA loaded on the SPs was measured using the Pierce BCA protein assay kit (Life Technologies SAS, Courtaboeuf, France). Briefly, $200 \mu 1$ of working reagent was added to $25 \mu \mathrm{L}$ of each sample in 96 well-plate. The absorbance at $562 \mathrm{~nm}$ was read on the Infinite ${ }^{\circledR} 200$ PRO microplate reader (TECAN Group Ltd., Mannedorf, Switzerland) after $30 \mathrm{~min}$ of incubation at $37{ }^{\circ} \mathrm{C}$ and cooling to RT for $10 \mathrm{~min}$. The concentration of the drug was extrapolated by a calibration curve prepared with different concentrations of rtPA.

The encapsulation efficacy (EE) was calculated as EE $(\%)=100 \% \times \mathrm{B} / \mathrm{A}$, whereas $\mathrm{B}$ is the amount of rtPA loaded onto the SPs and A is the total quantity of rtPA put in contact with the SPs.

In vitro rtPA release: The release of rtPA from the Fuco-SPs was assessed by flow cytometry.[44] FITC-rtPA (Abcam, Cambridge, United Kingdom) at $1 \mathrm{mg} / \mathrm{ml}$ was placed in contact with TRITC Fuco-SPs at $5 \mathrm{mg} / \mathrm{ml}$ for $1 \mathrm{~h}$ at RT. The suspensions were added to tubes pre-filled with $400 \mu \mathrm{L}$ of saline and placed under gentle agitation at $37^{\circ} \mathrm{C}$. At each time point of $0,15,30,45,60$, and 90 min, the tubes were analyzed with a BD FACS Aria'TM III flow cytometer (Becton Dickinson, New Jersey, United States). The TRITC-Dextran, excited by a $543 \mathrm{~nm}$ laser, was detected at $569 \mathrm{~nm}$, while the FITC-rtPA, excited at $480 \mathrm{~nm}$, was detected on a 530/30 nm PMT. Flow cytometry analyses were performed in triplicates with Diva software (Becton Dickinson). The protein release curve was obtained by normalizing the values of Mean Fluorescence Intensity (MFI) of the FITC-rtPA still associated with TRITC-Fuco-SPs. In vitro amidolytic activity of rtPA-loaded SPs: Amidolytic activity of rtPA loaded SPs was assessed with the fluorogenic substrate PefaFluor ${ }^{\circledR}$ tPA (Cryopep, Montpellier, France). $2.5 \mu \mathrm{L}$ of samples $(20 \mu \mathrm{g} / \mathrm{ml})$ was put in contact with $97.5 \mu \mathrm{L}$ of $100 \mathrm{mM}$ HEPES buffer $(\mathrm{pH}$ 8.0, $154 \mathrm{mM} \mathrm{NaCl}, 0.1 \% \mathrm{HAS})$ in the 96-well plate. After the addition of $10 \mu \mathrm{L} \mathrm{PefaFluor}{ }^{\circledR}$ at $1 \mathrm{mM}$, a kinetic profile was obtained by measuring the fluorescence level at $440 \mathrm{~nm}$ every 2 
min for 90 min at $37^{\circ} \mathrm{C}$ with Infinite ${ }^{\circledR} 200$ PRO microplate reader (TECAN Group Ltd., Mannedorf, Switzerland). Free rtPA was used at the same concentration based on the Pierce BCA protein assay. Increase of fluorescence corresponded to the fluorogenic peptide substrate hydrolysis by rtPA. Enzymatic activity was determined from the resulting kinetic profile and compared to the one of free rtPA.

In vitro fibrinolytic activity of rtPA-loaded SPs: To assess the fibrinolytic activity of rtPA-loaded SPs, a fibrin lysis clot experiment was performed. $5 \mathrm{~mL}$ of TRIS Buffer with 3\% w/v low melting agarose (Carl Roth GmbH p Co. KG, Karlsruhe, Germany) were heated to $65{ }^{\circ} \mathrm{C} .5 \mathrm{~mL}$ of fibrinogen (from human plasma, Sigma Aldrich) solution in TRIS buffer (5 $\mathrm{mg} / \mathrm{ml}$ ) was slowly heated to $37^{\circ} \mathrm{C}$. Once the agarose solution reached $65{ }^{\circ} \mathrm{C}$, it was cooled to $37^{\circ} \mathrm{C}$, and $2.5 \mathrm{U}$ of thrombin (from human plasma, Sigma Aldrich) was added. Next, a fibrinogen solution was slowly added into the agarose/thrombin mixture under gentle agitation to avoid the formation of bubbles. The reaction mixture was poured into a $9 \mathrm{~cm}$ Petri dish and cooled at $4{ }^{\circ} \mathrm{C}$ for $30 \mathrm{~min}$ until the fibrin clot became visible. On the solidified agarose gel, round wells were formed using a $3 \mathrm{~mm}$ punch as sample reservoirs. $5 \mu \mathrm{L}$ of each SPs sample (45 $\mu \mathrm{g} / \mathrm{ml}$ ) was dropped into the wells and incubated overnight at $37^{\circ} \mathrm{C}$ in a humid environment. The degree of fibrin lysis was quantified with ImageJ by comparing the size of the fibrinolysis circle of the samples and free rtPA at the equivalent concentration based on the Pierce BCA protein assay.

Flow microchamber experiments: An in vitro flow adhesion assay was performed to evaluate the affinity of the Fuco-SPs with their molecular target. Micro-channels of Vena8 Fluoro $^{+}$chambers (width: $0.04 \mathrm{~cm}$, height: $0.01 \mathrm{~cm}$, and length: $2.8 \mathrm{~cm}$; Cellix Ltd, Dublin, Ireland) were coated overnight with recombinant human P-selectin, L-selectin or E-selectin (R\&D systems France, Lille, France) at $100 \mu \mathrm{g} / \mathrm{ml}$ and left overnight at $4{ }^{\circ} \mathrm{C}$. To confirm the concentration-dependent binding of Fuco-SPs to P-selectin, some channels were coated with P- 
selectin at a range of concentrations $(5,25,50$, and $100 \mu \mathrm{g} / \mathrm{ml})$. Channels were then washed with $\mathrm{NaCl} 0.9 \%$ and further incubated with $\mathrm{HSA}$ at $10 \mu \mathrm{g} / \mathrm{ml}$ for $2 \mathrm{~h}$.

A suspension of fluorescently labeled Control-SPs or Fuco-SPs $(1 \mathrm{mg} / \mathrm{ml})$ in saline was passed through the channels for $5 \mathrm{~min}$ at arterial and venous flow conditions (shear stress 67.5 dyne $/ \mathrm{cm}^{2}$ and 6.75 dyne $/ \mathrm{cm}^{2}$ ) using an ExiGo ${ }^{\mathrm{TM}}$ pump (Cellix Ltd, Dublin, Ireland). For the competitive binding experiment, fucoidan solution $(10 \mathrm{mg} / \mathrm{ml})$ was injected $5 \mathrm{~min}$ prior to the Fuco-SPs at the same rate. Then, all the channels were washed with $\mathrm{NaCl} 0.9 \%$ for $1 \mathrm{~min}$. The binding of the adhered SPs was visualized in real-time under fluorescence microscopy (Axio Observer, Carl Zeiss Microimaging GmbH, Iena, Germany). For the quantitative analysis, the number of fluorescent SP clusters on each channel was measured using the "Analyze particles" tool in the image analysis software ImageJ (NIH, Bethesda, U.S.) with a 4-pixel threshold to eliminate the background noise.

To further investigate the binding efficiency of unloaded and loaded SPs to activated platelets, the microchannels of Vena8 Fluoro ${ }^{+}$were coated with fibrillar type I collagen Horm ${ }^{\circledR}$ (50 $\mu \mathrm{g} / \mathrm{ml}$ ) overnight at $4{ }^{\circ} \mathrm{C}$ and rinsed with $\mathrm{NaCl} 0.9 \%$ before use. Human whole blood (EFS, Bichat Hospital, Paris, France), collected in the PPACK tubes and labeled with $5 \mu$ M DIOC6 (Life Technologies SAS, Saint-Aubin, France), was perfused at arterial shear stress for 5 min to induce platelet activation and aggregation. Platelet aggregation through contact with collagen was visualized in real-time with phase-contrast microscopy (Axio Observer, Carl Zeiss Microscopy, Oberkochen, Germany). After rinsing with $\mathrm{NaCl} 0.9 \%$, fluorescent Control-SPs or Fuco-SPs (unloaded or loaded with rtPA) at $1 \mathrm{mg} / \mathrm{ml}$ were injected into the channels in saline for $5 \mathrm{~min}$. Their accumulation onto activated aggregates was monitored in real-time. Channels were then washed for $1 \mathrm{~min}$ with $\mathrm{NaCl} 0.9 \%$. Finally, the MFI of the fluorescent SPs bound to the platelets on each channel was analyzed with ImageJ. Intensity settings were kept the same for both types of SPs. 
Tissue distribution of Fuco-SPs in vivo: Animal studies were performed on C57BL/6

male mice (EJ, Le Genest, St-Berthevin, France) aged 5-8 weeks ( $25 \mathrm{~g}$ weight) in respect of the principles of laboratory about animal experimentation and with the approval of the animal care and use committee of the Claude Bernard Institute (APAFIS \#8724, Paris, France). Mice were anesthetized under the application of $2 \%$ isoflurane (Aerrane, Baxter). $200 \mu \mathrm{L}$ of FucoSPs $(5 \mathrm{mg} / \mathrm{ml})$ were injected through the retro-orbital route $(\mathrm{n}=3)$. To histologically analyze the particle accumulation, mice were sacrificed 30 min following their administration. The liver, spleen, lungs, and kidneys were excised, washed in saline, and fixed in paraformaldehyde $4 \%$. The tissues were then frozen and cryosectioned at $10 \mu \mathrm{m}$ thickness. The samples were stained with alcian blue \& nuclear fast red staining protocol. Strongly negative structures, including the Fuco-SPs, are stained blue, nuclei are stained pink to red, and cytoplasms appear pale pink. The slides with tissue slices were scanned with the Nanozoomer (Hamamatsu, Hamamatsu City, Japan) and viewed with the NDP.view2 software.

Animals and thromboembolic stroke model in vivo: Animal experiments were carried out on male Swiss wild-type mice (8-9 weeks old; 35-45 g; CURB, Caen, France). All experiments were performed following the French (Decree 87/848) and the European Communities Council (2010/63/EU) guidelines and were approved by the institutional review board (French ministry of Research). All the experiments were validated by the local ethical committee of Normandy (CENOMEXA) registered under the reference number APAFIS\#13172. Anesthesia was induced by the application of 5\% isoflurane (Aerrane, Baxter) and maintained by $2 \%$ isoflurane in a mixture of $\mathrm{O}_{2} / \mathrm{N}_{2} \mathrm{O}(30 \% / 70 \%)$.

Mice were placed in a stereotaxic device, then a small craniotomy was performed, the dura was excised, and the middle cerebral artery (MCA) was exposed. To induce the occlusion of the MCA, the coagulation cascade was triggered by the pneumatical injection of $1 \mu \mathrm{L}$ murine $\alpha$-thrombin (1 IU; Stago BNL) with a glass micropipette, as previously described.[73] 
Successful MCA occlusion was confirmed by the Laser Doppler flowmeter (Oxford Optronix).

For the treatment, the animals were intravenously injected through a tail vein catheter $(200 \mu \mathrm{L}$, $10 \%$ bolus, $90 \%$ infusion over 40 minutes) with either saline, Actilyse ${ }^{\circledR} \mathrm{rtPA} 10 \mathrm{mg} / \mathrm{kg}$, or rtPAFuco-SPs (loaded rtPA at $10 \mathrm{mg} / \mathrm{kg}$, SPs at $71 \mathrm{mg} / \mathrm{kg}$ ) 20 minutes after thrombus formation (n $\geq 5$ ). Brain perfusion was monitored by Laser Speckle Contrast Imager (MOOR FLPI-2, Moor Instruments) throughout the treatment. Region of interest (ROI) was selected on the ipsilateral to occlusion and contralateral hemispheres to monitor the relative cerebral blood flow in the affected region $\mathrm{Fl}_{\mathrm{t}}(\%)=100 \% \mathrm{x} \mathrm{Fl}_{\mathrm{ipsi}} / \mathrm{Fl}_{\text {contra. }}$. The post-stroke reperfusion was expressed as a Growth Rate (GR) of the blood flow increase in the ipsilateral ROI to contralateral one at a time point, and it was quantified as GR $(\%)=100 \% \times\left(\mathrm{Fl}_{\mathrm{t} 2}-\mathrm{Fl}_{\mathrm{t} 1}\right) / \mathrm{Fl}_{\mathrm{t} 1}$

Magnetic resonance imaging acquisition and analysis: Mice were anesthetized with 5\% isoflurane and maintained with 1.5-2\% isoflurane in a mixture of $\mathrm{O}_{2} / \mathrm{N}_{2} \mathrm{O}(30 \% / 70 \%)$ during the acquisitions. Experiments were carried out on a Pharmascan 7T (Bruker Biospin, Wissembourg, France). Three-dimensional T2-weighted images were acquired using a MultiSlice Multi-Echo sequence (TE/TR $39.9 \mathrm{~ms} / 3,500 \mathrm{~ms}, 2$ averages) $24 \mathrm{~h}$ after the stroke. Lesion volumes were quantified on these images using ImageJ software (slice thickness $0.5 \mathrm{~mm}$ ). Magnetic resonance angiography was performed using a 2D-TOF sequence (TE/TR $4.24 \mathrm{~ms} /$ $12 \mathrm{~ms}, 1$ average) $24 \mathrm{~h}$ after ischemia, and the recanalization status of the MCA was determined blindly from the analysis of the merged MCA angiograms with maximum intensity. The angiographic score is based on the TICI (Thrombolysis in Cerebral Infarction) grade flow scoring (from Score 0: no perfusion to Score 3: full recanalization). For the in vivo detection of the BBB permeability, three dimensional T1 FLASH sequences (TE/TR $4.37 \mathrm{~ms} / 15.12 \mathrm{~ms} ; 3$ averages) were used, $15 \mathrm{~min}$ after the intravenous injection of $200 \mu \mathrm{L}$ gadolinium chelate (DOTAREM) in saline $(0.25 \mathrm{mg} / \mathrm{ml})$. BBB leakage was measured 4 days after the stroke induction, and its volume was quantified using ImageJ. 
Thrombus targeting by Fuco-SPs in a murine model of venous thrombosis: Animal studies were done following principles of laboratory animal care and with the approval of the animal care and use committee of the Claude Bernard Institute (APAFIS \#8724, Paris, France). $\mathrm{FeCl}_{3}$-induced in vivo thrombosis model on mesenteric vein was carried out on $\mathrm{C} 57 \mathrm{BL} / 6$ male mice (EJ, Le Genest, St-Berthevin, France) aged 5-8 weeks. Mice were anesthetized with an intraperitoneal injection of ketamine $(100 \mathrm{mg} / \mathrm{kg})$ and xylazine $(10 \mathrm{mg} / \mathrm{kg})$. After midline abdominal incision, the mesentery was exposed, and vessels were visualized by an intravital microscope (Leica MacroFluo, Leica Microsystems SAS, Nanterre Cedex, France) using Orca Flash 4.0 scientific CMOS camera (Hamamatsu Photonics France SARL, Massy, France). For green fluorescent labeling of mitochondria of platelets and leucocytes, DIOC6 (Life Technologies SAS, Saint-Aubin, France) at $25 \mu \mathrm{M}$ was retro-orbitally injected. The mesentery vein was covered with a $1 \mathrm{~mm}$ Whatman chromatography paper that was prior soaked in a $10 \%$ w/v iron chloride (Sigma-Aldrich) solution for $1 \mathrm{~min}$, and then washed with saline. The formation of non-occlusive thrombi was monitored in real-time by fluorescence microscopy by an accumulation of fluorescently labeled platelets. TRITC-fluorescent labeled Control-SPs or Fuco-SPs were retro-orbitally injected $10 \mathrm{~min}$ after thrombus initiation with the volume of 150 $\mu \mathrm{L}$ (5 mice per group).

For histological evaluation, mice were sacrificed with pentobarbital overdose $5 \mathrm{~min}$ after administration of SPs. The affected part of the mesenteric vein was cut, washed in $0.9 \%$ $\mathrm{NaCl}$, fixed in paraformaldehyde $4 \%(\mathrm{w} / \mathrm{v})$, and frozen. The vein samples were cryosectioned at $10 \mu \mathrm{m}$ thickness. The cell nuclei of a venous vascular wall were labeled with DAPI (Thermo Fisher Scientific, Massachusetts, United States) contained in a mounting medium (Vecto laboratories). The samples were observed by fluorescence microscopy. For the quantitative analysis, normalized MFI of the TRITC signal from SPs was expressed, defined as total TRITC 
fluorescence intensity divided by the size of the thrombus area on 2 slides from each mouse with the ImageJ (NIH, Bethesda, U.S.).

Statistical analysis: Quantitative data were expressed as mean \pm standard error of the mean $(\mathrm{SEM})(\mathrm{n} \geq 3)$. Statistical tests were carried out with GraphPad Prism 9 (GraphPad Software, Inc., La Jolla, U.S.) with a 95\% confidence level. Kolmogorov-Smirnov normality test was utilized to examine if variables are normally distributed. Normally distributed data were analyzed then with unpaired t-test or one-way analysis of variance (ANOVA) with post hoc Turkey's test. The Mann-Whitney U test was applied otherwise. The p-values of $* \mathrm{p}<0.05$; $* * \mathrm{p}<0.01 ; * * * \mathrm{p}<0.001$ were considered statistically significant.

\section{RESULTS}

\subsection{Submicroparticle synthesis and characterization}

Novel polysaccharide SPs were elaborated by a simple and reproducible two-step synthesis process (Figure 1A). First, a stable w/o miniemulsion of the aqueous phase with hydrophilic polysaccharides and vegetable (sunflower) oil was prepared. Chemical crosslinking of polysaccharides with the crosslinking agent STMP under alkaline conditions (Figure 1B) produced a suspension of uniform SPs. We refer to the hydrogel particles only from dextran as Control-SPs, and from a mixture of dextran and fucoidan as Fuco-SPs in this manuscript. 
A
B
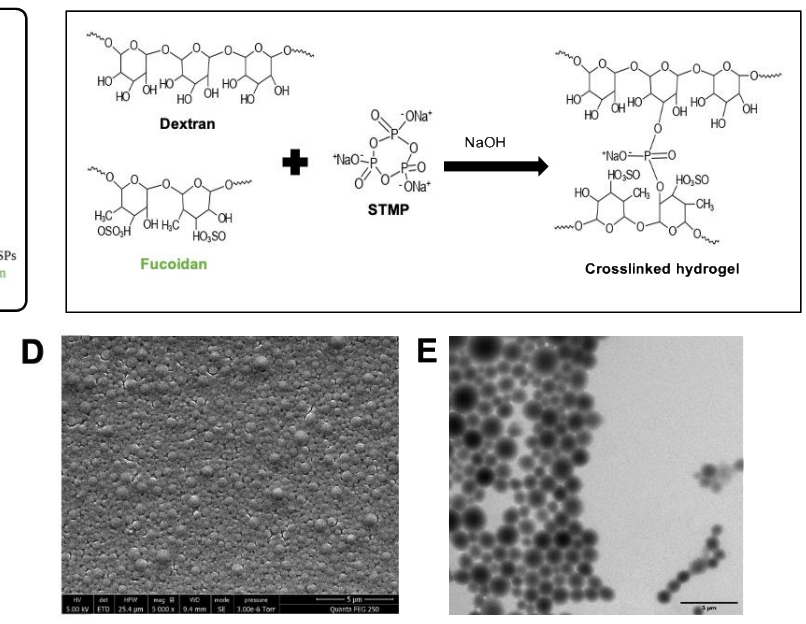

G
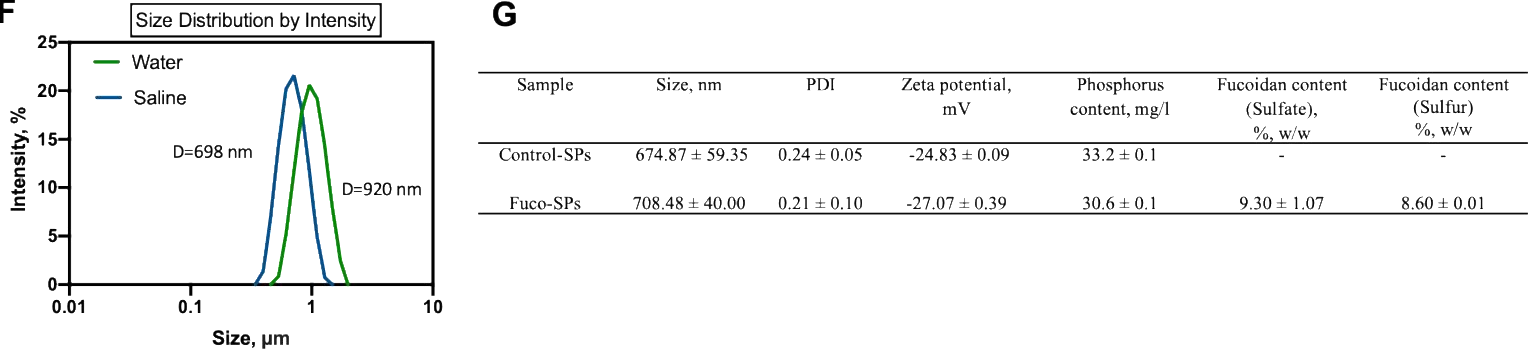

Figure 1. Synthesis and physico-chemical characterization of the SPs. A. Overall schematic of the synthesis process of the SPs - miniemulsion / crosslinking. B. Crosslinking of the polysaccharides (dextran and fucoidan) with STMP in alkaline conditions. C. Proposed therapeutic mode of action of the rtPA-loaded Fuco-SPs after the ischemic stroke: the FucoSPs accumulate on the surface of the activated platelets due to P-selectin affinity of fucoidan and perform local thrombolysis with alteplase. SEM (D) and TEM (E) images of the Fuco-SPs. F. Swelling in the water of the Fuco-SPs due to the hydrogel nature of the particles. The size of one sample when resuspended in water (green) and in saline (blue). G. Size, zeta potential, and chemical composition of the SPs.

To ensure desirable safety of the future drug delivery platform, a thoughtful approach to material selection was effectuated. Low molecular weight dextran $40 \mathrm{kDa}$ of clinical-grade was utilized without any chemical modification. Having a large number of hydroxyl groups, dextran is a suitable compound for subsequent chemical crosslinking with STMP,[28] an FDAapproved food additive[31] that is preferred over conventional crosslinker glutaraldehyde known for cytotoxicity.[32] Fucoidan, a marine polysaccharide approved as a pharmaceutical compound[33] that exhibits a nanomolar affinity to P-selectin,[34] served as a targeting ligand to thrombi. Hence, both natural polysaccharides applied in this study are affordable, 
biodegradable, biocompatible compounds, non-immunogenic, and approved for clinical applications.

Instead of commonly used organic solvents, sunflower oil was applied as an emulsion continuous phase. The choice of the stabilizing agent plays an important role in reducing the interfacial tension and Laplace pressure when fabricating a stable emulsion and future nanocarrier. In this work, we selected a potent oil-soluble nonionic surfactant for stabilizing w/o emulsions - PGPR, which is also recognized by the FDA as a safe compound and is frequently used as an emulsifier in the food production industry.[35] In addition, to counteract the Ostwald ripening of water droplets, $6 \mathrm{M} \mathrm{NaCl}$ was added to the aqueous phase as an osmotic agent to adjust the osmotic gradient and to stabilize the w/o emulsion further. Overall, multiple parameters were optimized to obtain a stable and homogenous miniemulsion and subsequent nano-delivery system. It was found that the size of the templating droplet and the ultimate hydrogel SPs being directly proportional to the polysaccharide molecular weight and inversely proportional to the amount of surfactant and crosslinking agent as well as homogenization speed (data not shown).

ESEM and TEM images revealed a well-defined spherical morphology and uniform size distribution of SPs (Figure 1D, E). As hydrogel-based particles, they could swell in an aqueous medium while maintaining their network structure (Figure 1F).

Functionalized Fuco-SPs contained $8.60 \pm 0.01 \%$ of fucoidan in a mass of the total SPs weight, determined by elemental analysis of sulfur, and $9.30 \pm 1.07 \%$ of fucoidan by quantification of the sulfate content by a semi-quantitative colorimetric assay. In such a way, two different techniques estimated $\sim 9 \%$ fucoidan composition in the SPs. The physicochemical properties of these nanoformulations are displayed in Figure 1G. The SPs exhibited the hydrodynamic size $674.87 \pm 59.35 \mathrm{~nm}$ (Control-SPs) and $708.48 \pm 40.00 \mathrm{~nm}$ (Fuco-SPs), determined by DLS. It is important to highlight that a relatively large size of the SPs might limit 
the accumulation of associated rtPA within the brain parenchyma and reduce the risk of hemorrhagic events. The negative $\zeta$-potential of the SPs $-24.83 \pm 0.09 \mathrm{mV}$ for Control-SPs and $-27.07 \pm 0.39 \mathrm{mV}$ for Fuco-SPs ensured colloidal stability as a result of the anionic nature of the fucoidan and the formation of the anionic phosphate functional groups, produced during the crosslinking reaction with STMP. The phosphorus content of the SPs is indicated in Figure $1 \mathrm{G}$.

The size and zeta potential of both SPs remained relatively stable for at least 30 days at $4{ }^{\circ} \mathrm{C}$. Besides, effective storage of the SPs can be ensured by freeze-drying with $5 \%(\mathrm{w} / \mathrm{v})$ sucrose as a cryoprotectant and subsequent resuspension in an aqueous medium. The overall yield of the synthesis was $13.4 \pm 0.7 \mathrm{mg}$ of SPs.

\subsection{Biocompatibility of the SPs}

The injectable hydrogel SPs were produced according to the green chemistry principles through the formulation method without using hazardous substances and organic solvents and were expected to be biocompatible.

An initial in vitro evaluation of biocompatibility of the developed SPs examined cytoand hemocompatibility. The cytocompatibility of the SPs was assessed with a resazurin cell viability assay on endothelial cells. Following $24 \mathrm{~h}$ exposure, Control-SPs and Fuco-SPs did not affect cellular viability and metabolic activity of HUVECs at concentrations ranging from 0.1 to $1.5 \mathrm{mg} / \mathrm{ml}$, exhibiting an excellent cytocompatible profile (cell survival $>90 \%$, up to the highest tested concentrations of SPs) (Figure 2A). The upper limit for the tested concentration $1.5 \mathrm{mg} / \mathrm{ml}$ of the SPs was selected to surpass the tested concentrations for the majority of the nanosystems in vitro (typically, maximum $400 \mu \mathrm{g} / \mathrm{ml}$ )[36] and the concentration of the SPs employed for further in vivo experiments in this work $(71 \mathrm{mg}$ SPs per $1 \mathrm{~kg}$ body weight or 1.1 mg SPs per $1 \mathrm{~mL}$ of blood). There was no significant difference between the Control-SPs and Fuco-SPs, as both did not provoke cytotoxicity. 
A

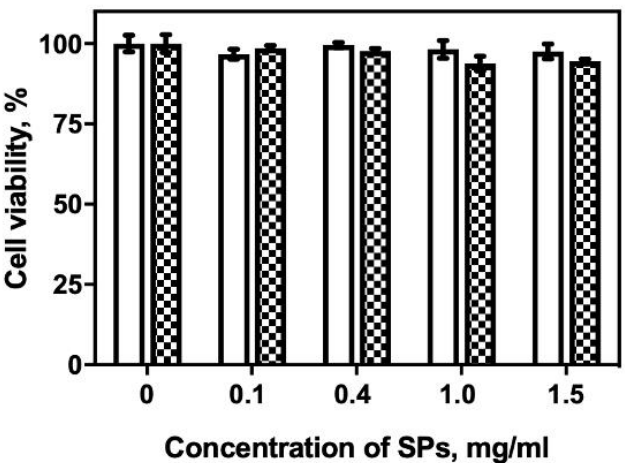

C
B

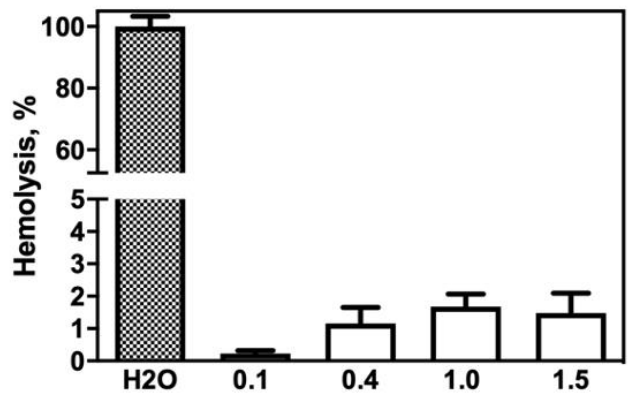

Concentration of Fuco-SPs, mg/ml
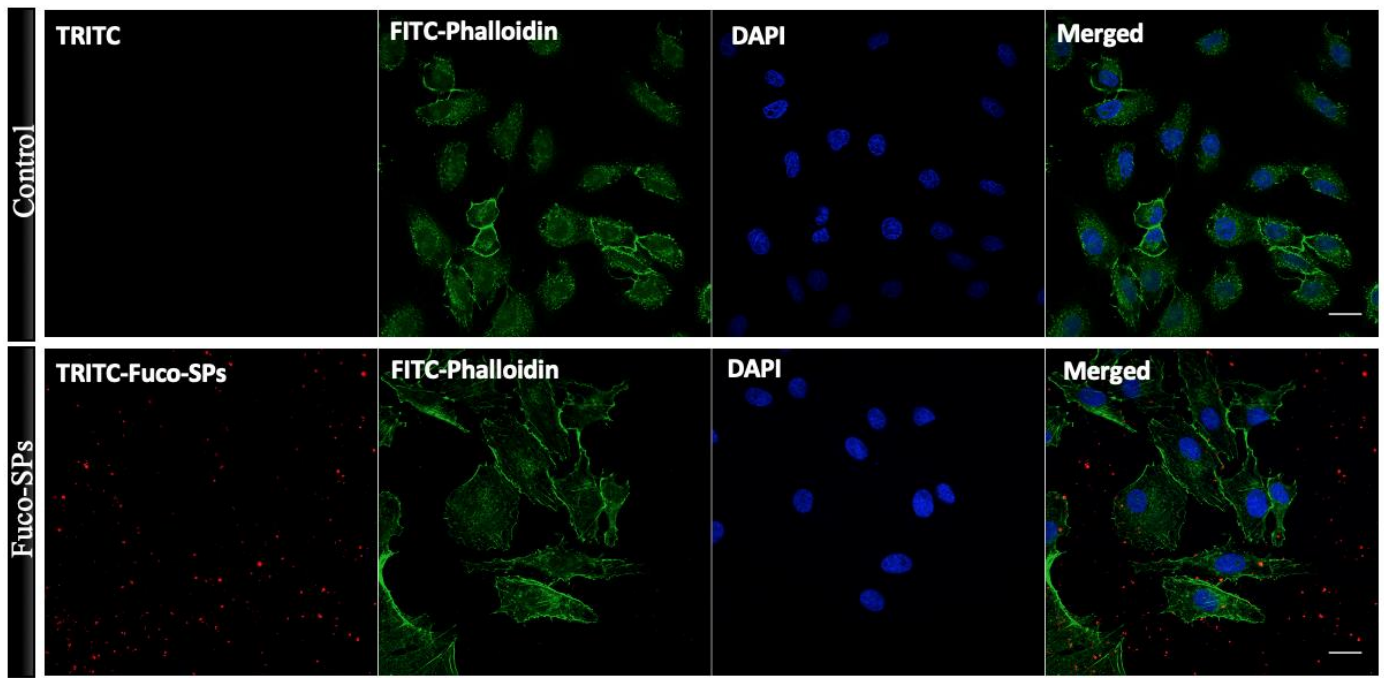

Figure 2. Biocompatibility of the SPs. Cytocompatibility (A) and hemocompatibility (B). Confocal microscopy of HUVECs cultured without (control) and with Fuco-SPs (C) (scale bar $=30 \mu \mathrm{m})$.

Since the SPs in this study were designed for intravenous administration and were expected to have direct contact with blood, the Fuco-SPs were examined for their bloodcompatible behavior by a hemolysis test on isolated murine red blood cells in vitro (Figure 2B). Even at the highest concentration of $1.5 \mathrm{mg} / \mathrm{ml}$, the SPs presented a hemolytic index $1.51 \pm$ $0.02 \%$, below $2 \%$, and considered nonhemolytic according to ISO 10993 - 4 standard.[37,38]

Morphology of the cells, co-cultured with Fuco-SPs, was visualized with confocal microscopy. No obvious morphological differences were revealed for HUVECs with Fuco-SPs and negative control, as depicted in Figure 2C. FITC-Phalloidin staining was used to visualize a cytoplasm and DAPI for nuclei. Moreover, the SPs were internalized by endocytosis as the 
merged images of all three probes revealed colocalization of the particles within the dye in the cytoplasm.

Collectively, these results suggest that the polysaccharide SPs have favorable biocompatibility for their application in vivo.

\subsection{Binding of SPs to P-selectin in vitro}

Knowing that fucoidan was homogeneously distributed in the structure of the hydrogel FucoSPs and constituted $\sim 9 \% \mathrm{w} / \mathrm{w}$ of the composition, we investigated whether its quantity on the surface was sufficient for specific adhesion to its molecular target. While most of the publications assess targeting strategy in vitro in static conditions by flow cytometry or confocal microscopy,[17,39,40] our group developed a robust and tunable dynamic microfluidic method that mimics arterial or venous blood flow conditions to study the targeting efficacy for recombinant $\mathrm{P}$-selectin or/and human activated platelet aggregates expressing $\mathrm{P}$-selectin. It was previously validated on fucoidan-coated nano-/microcarriers (Figure S1, Supplementary Data).[18-20]

First, fluorescent Fuco-SPs and Control-SPs were injected in the microchannels coated with recombinant P-selectin under arterial or venous shear rates $\left(67.5\right.$ dyne $\mathrm{cm}^{-2}$ vs. 6.75 dyne $\mathrm{cm}^{-2}$ ), and their adhesion was visualized and quantified in real-time under fluorescence microscopy. According to obtained results, fluorescent Fuco-SPs depicted a significantly higher adhesion to P-selectin coating than Control-SPs both in arterial (374.25 \pm 115.33 adhered FucoSPs vs. $30.25 \pm 13.84$ adhered Control-SPs, $\left.{ }^{*} \mathrm{p}<0.05\right)$ and venous $(228.25 \pm 36.67$ adhered Fuco-SPs vs. $34.50 \pm 18.16$ adhered Control-SPs, $* *$ p <0.01) flow conditions (Figure 3A, E). There was no significant difference between the fluorescent signal of Fuco-SPs accumulation for arterial and venous flow conditions $(\mathrm{p}=0.2731)$. Fuco-SPs accumulation after injection onto P-selectin coating was in a linear dose-dependent manner as regards to the P-selectin 
concentration, $\mathrm{R}^{2}=0.9904$ (Figure 3B). An experiment of competitive interaction illustrates that fucoidan solution pre-injection at $10 \mathrm{mg} / \mathrm{ml}$ considerably reduced the attachment of the FucoSPs onto the microchannels with P-selectin $(374.25 \pm 115.33$ vs. $19.75 \pm 10.06, * \mathrm{p}<0.05)$ (Figure 3C).
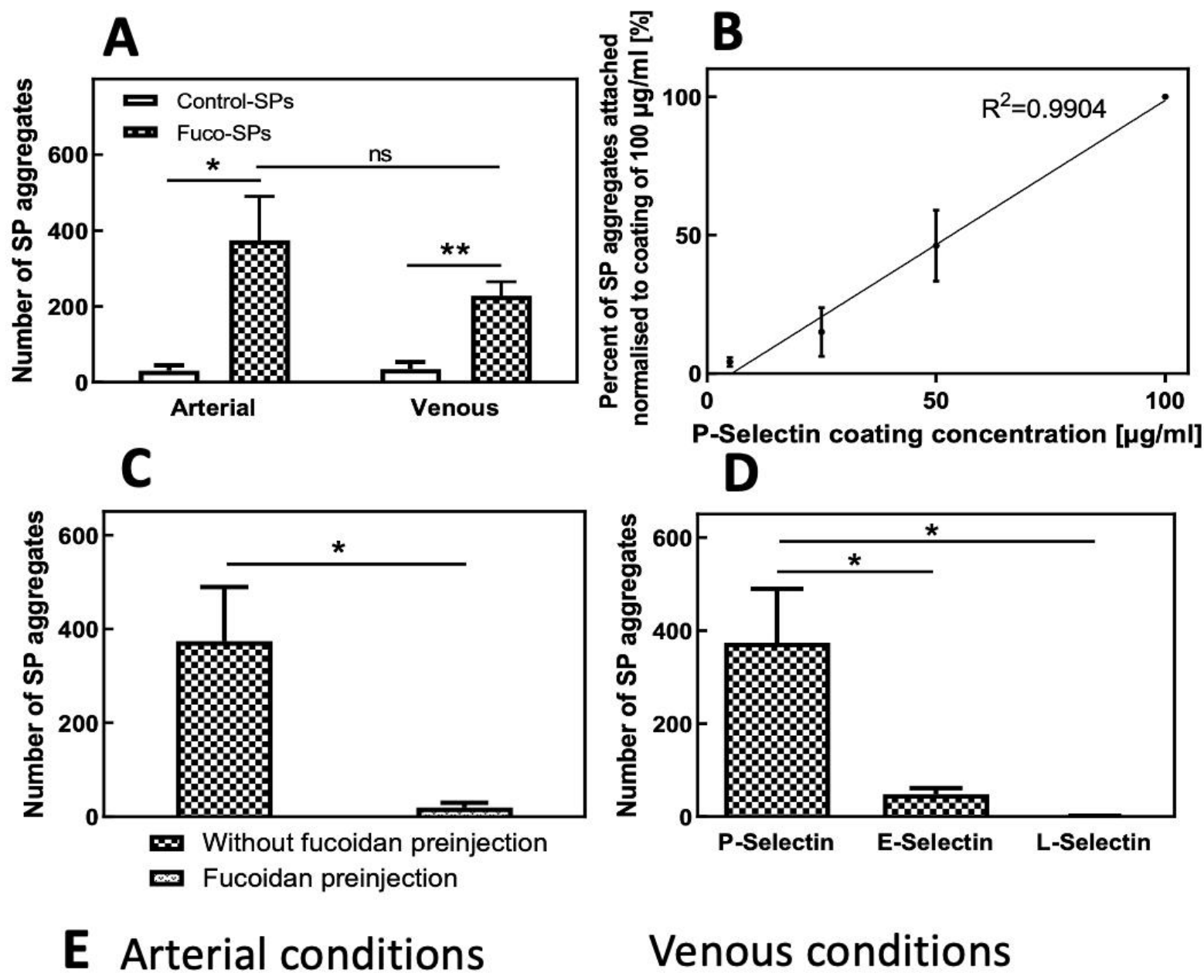

\section{Venous conditions}
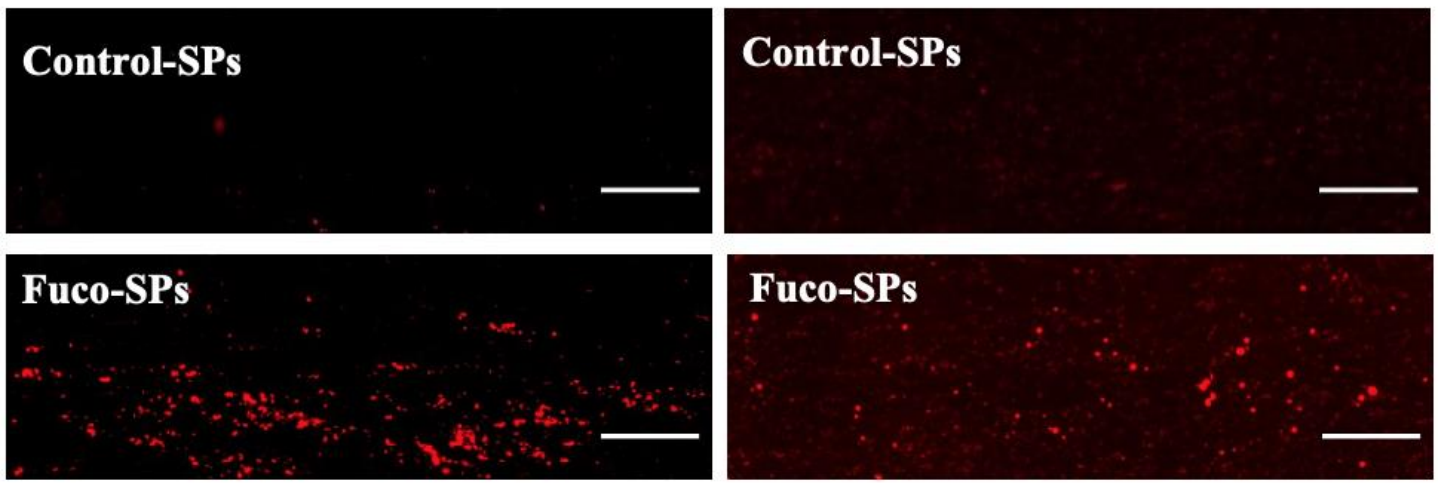

Figure 3. Evaluation of the SPs interactions with selectins. A. Adhesion of the Control-SPs or Fuco-SPs on the coating of the recombinant P-selectin in the microfluidic assay under arterial and venous flow conditions $(n=4)$. B. Concentration-dependent binding of the Fuco-SPs onto the coating of the P-selectin at a range of concentrations. C. Fucoidan pre-injection inhibited 
Fuco-SPs adhesion onto the P-selectin. D. Comparison of the Fuco-SPs binding to other selectins: E- and L-Selectin. E. Fluorescent microscope view of Control-SPs or Fuco-SPs adhesion over a P-selectin coating of $100 \mu \mathrm{g} / \mathrm{ml}$ under arterial and venous shear rates (scale bar $=20 \mu \mathrm{m})$.

To establish the specificity of the Fuco-SPs binding to P-selectin, the targeting assay was extended to other members of the selectin family: E- and L-selectin.[41] The percentage of the Fuco-SPs adhered to the E- and L-selectin was normalized over the mean number of the attached Fuco-SPs to a P-selectin coating at the equivalent concentration. Indeed, only $12.73 \pm$ $3.66 \%$ of the SPs adhered to E-selectin and $0.26 \pm 0.19 \%$ to L-selectin coating (Figure 3D). Thus, our results indicate that Fuco-SPs bind specifically to P-selectin but not to E- and Lselectins, and these results are in accordance with a previous work of our group published by Bo L. et al. of fucoidan-functionalized polymer microcapsules.[18]

Overall, these findings are encouraging evidence of the sensitivity and selectivity of the Fuco-SPs, confirming fucoidan potential as a natural ligand of P-selectin.

\section{4.rtPA loading onto the SPs and its release in saline. In vitro thrombolytic activity o rtPA-loaded SPs}

Due to rtPA low bioavailability and requirement of high dose administration, coupling this enzyme to the biocompatible nanocarrier could overcome the drawbacks associated with a drug high dosage. Herein, an efficient rtPA encapsulation was achieved through the physical adsorption method due to electrostatic interaction: the protein, which is an amphoteric molecule, was put in contact with negatively charged polysaccharide SPs in the water at $\mathrm{pH}$ below rtPA isoelectric point IP=7.7[42,43] when rtPA presented a positive charge. The hydrogel nature of the SPs allowed reaching a high encapsulation efficiency of the rtPA of $64.78 \pm 2.16 \%$ and $81.04 \pm 1.86 \%$ for Control-SPs and Fuco-SPs, respectively. The confocal microscopy images of FITC-rtPA loaded onto TRITC-labelled Fuco-SPs revealed the uniform distribution of the rtPA within a porous structure of the hydrogel SPs, as evidenced by a green fluorescence from 
FITC-rtPA colocalized with the red fluorescence from the particles (Figure S2A, Supplementary Data).

The release kinetics of rtPA from fucoidan-functionalized SPs was analyzed in vitro by flow cytometry[44] in saline at $37{ }^{\circ} \mathrm{C}$ under gentle agitation by quantifying the MFI of the FITC-labelled rtPA associated with TRITC-fluorescent Fuco-SPs. Figure S2B, Supplementary Data indicated a gradual and continuous sustained release of the lytic agent from the SPs during the observation period: $46.41 \pm 1.34 \%$ of the encapsulated protein was released during the first $15 \mathrm{~min}$, and $76.98 \pm 1.74 \%$ after $90 \mathrm{~min}$. This release profile is classical for the hydrogels.[45]

The thrombolytic activity of the rtPA-loaded SPs in vitro was analyzed as a combination of amidolytic and fibrinolytic activities (Figure 4A, B, C). Amidolytic or enzymatic activity featured the ability of the proteolytic enzyme to hydrolyze the rtPA substrate. Interestingly, the amidolytic activities of rtPA on Control-SPs and Fuco-SPs were comparable to that of free rtPA (Figure 4A \& 4B). The fibrinolytic experiment in vitro of the rtPA-loaded SPs was performed in a fibrin plate assay (Figure S3A, Supplementary Data). The results indicated full retention of fibrinolytic activity (Figure 4C; Figure S3B, Supplementary Data). rtPA loaded onto the SPs appeared to diffuse into the fibrin-agarose matrix and induce fibrinolysis in contact with fibrin. No significant difference was detected between both types of SPs, enabling us to utilize them in the following experiments.

Overall, the rtPA association with the SPs did not affect the drug amidolytic activity and fibrinolytic potential in our design. This result is in accordance with most studies on nanogels suggesting that drug encapsulation via a passive diffusion into the preformed nanogels does not affect the secondary structure of the adsorbed protein and its biological activity.[46] 

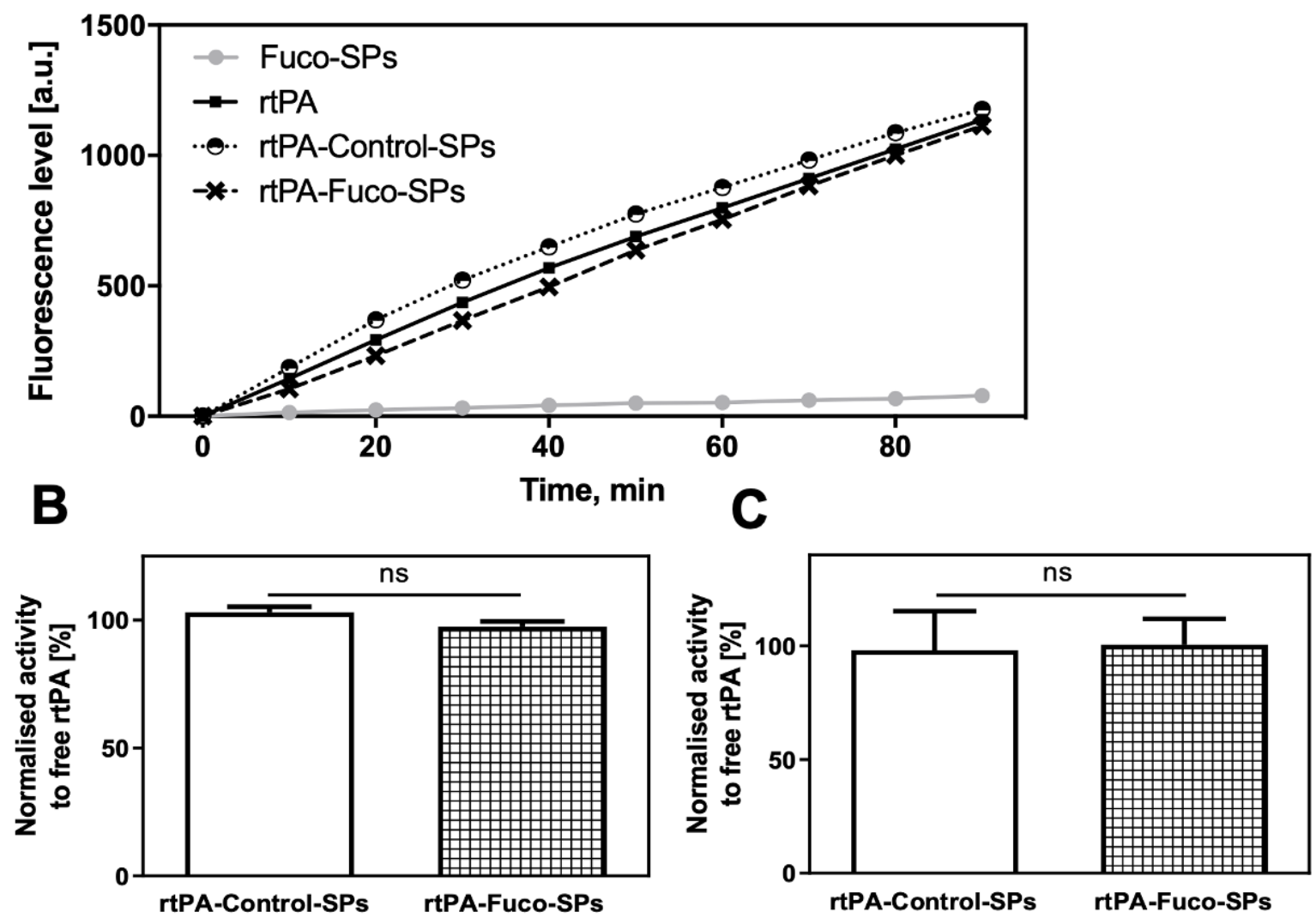

Figure 4. Thrombolytic efficacy in vitro of the rtPA-loaded SPs. A - B. Amidolytic activity measured by the PefaFluor ${ }^{\circledR}$ fluorogenic assay. A. The curves correspond to the mean value of fluorescence release and are correlated to the enzymatic velocity over $90 \mathrm{~min}$. B. Corresponding quantitative analysis normalized to free $\mathrm{rtPA}$ at the same concentration at $90 \mathrm{~min}(\mathrm{n}=3)$. C. Fibrinolytic activities of the SPs determined by a fibrin-plate agarose assay. The quantitative analysis normalized to free rtPA at the same concentration $(n=3)$.

\subsection{Unloaded and rtPA-loaded Fuco-SPs adhere to activated platelet aggregates in vitro}

\section{under arterial flow}

Since aggregation of activated platelets and platelet-mediated coagulation pathways are hallmark events in thrombosis, activated platelets are a suitable cellular target for nanocarrier binding to thrombi.[47] Before the in vivo tests, we complemented the targeting evaluation on selectins with the second set of microfluidic experiments to validate Fuco-SPs capability to actively anchor onto the surface of activated platelets, expressing P-selectin. Thus, human whole blood was passed into collagen-coated microchannels to induce platelet activation and aggregation. Fuco-SPs or Control-SPs were then perfused at arterial shear stress and the 
accumulation of the fluorescence from the adhered SPs was detected on the surface of activated

platelet aggregates. A quantitative analysis of the MFI revealed that Fuco-SPs adhered significantly more onto activated platelets than Control-SPs (2,678.34 \pm 237.40 for Fuco-SPs vs. $392.44 \pm 137.15$ for Control-SPs, $* * * \mathrm{p}<0.001$, Figure 5A \& 5B Left). Notably, adsorption of rtPA did not impair the Fuco-SPs clot-binding ability $(1,880.80 \pm 429.37$ for rtPA-Fuco-SPs vs. $77.56 \pm 40.25$ for rtPA-Control-SPs, ${ }^{* *} \mathrm{p}<0.01$, Figure 5B Right). There was no significant difference between unloaded and rtPA-loaded Fuco-SPs adhering to the activated platelets $(\mathrm{p}=0.1149)$.

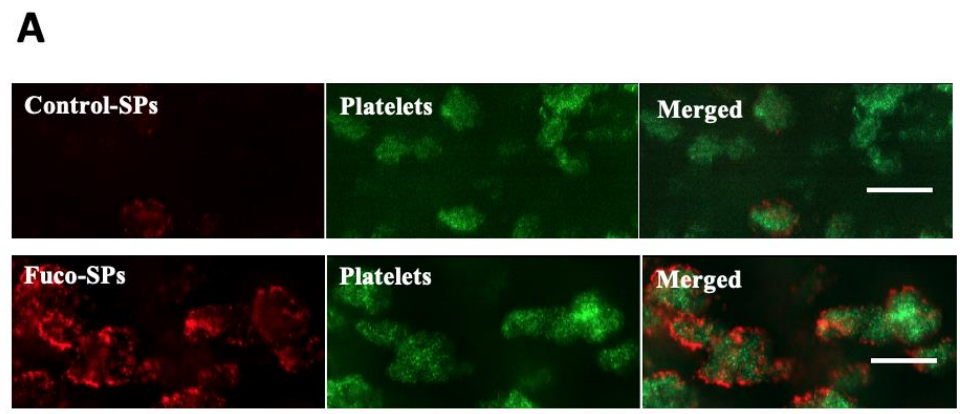

B

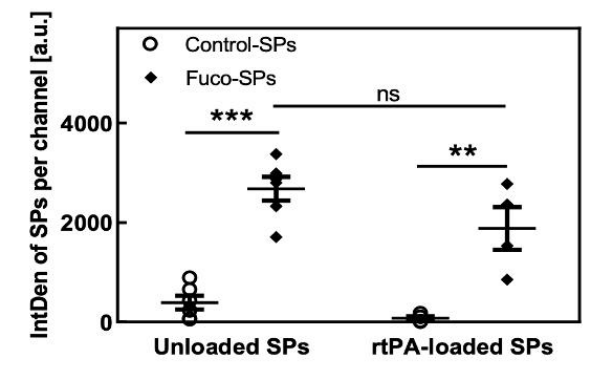

Figure 5. Adhesion of the SPs over activated platelet aggregates. A. Visualization by fluorescent microscopy of the attached unloaded SPs on the microchannels after the formation of the platelet aggregates (scale $\mathrm{bar}=20 \mu \mathrm{m}$ ). B. Corresponding quantification of the integral density of the unloaded $(n=6)$ and rtPA-loaded $(n=4)$ Control-SPs and Fuco-SPs in ImageJ.

In conclusion, these in vitro experiments provided crucial evidence of molecular interaction and high affinity between the P-selectin on the activated platelets and fucoidanfunctionalized SPs, which was maintained after loading the thrombolytic agent. This finding presumes that the administration of the rtPA-Fuco-SPs could enable a specific delivery of the rtPA-immobilized SPs to the platelet-rich thrombus with higher drug accumulation.

\subsection{Tissue distribution in vivo of fucoidan-functionalized SPs}

After their intravenous injection, the distribution in vivo of Fuco-SPs was examined by histological analysis of the excised tissues with alcian blue staining of negatively charged 
particles in the healthy mouse. The presence of Fuco-SPs in four main organs of excretion (liver, spleen, lungs, and kidneys) was assessed on several sections for each organ. Our results revealed that the polysaccharide Fuco-SPs distributed primarily into the spleen postadministration, indicating the splenic clearance. The particles accumulated in the liver to a lower extent, while their presence in the kidneys and lungs remained minor. These differences are presented in Figure S4, Supplementary Data. Our data are in agreement with other deformable polymer hydrogel-like particles in their submicron and micron size range.[48]

\subsection{In vivo thrombolytic efficacy}

Whereas demonstrating the in vitro activity of the rtPA immobilized on the drug delivery system is important, the in vivo therapeutic effect is paramount. A murine thromboembolic stroke model was established by in situ injection of 1 IU of thrombin into the MCA by provoking a coagulation cascade and formation of both fibrin- and platelet-rich clots in the lumen of the artery.[49,50] Figure 6A highlights the in vivo experiments design, and Figure 1C the potential mode of action of the Fuco-SPs on the thrombus. The treatment options control saline, rtPA $10 \mathrm{mg} / \mathrm{kg}$, or rtPA-Fuco-SPs at $10 \mathrm{mg} / \mathrm{kg}$ - were intravenously injected 20 min after ischemic onset under rtPA clinical mode of administration: $10 \%$ bolus followed by $90 \%$ infusion. It is important to note that $10 \mathrm{mg} / \mathrm{kg}$ is a relevant dose in mice in place of 0.9 $\mathrm{mg} / \mathrm{kg}$ in humans because of a lower sensitivity of human rtPA in murine plasma.[51] No morbidity or mortality was observed in the mice during therapeutic experiments, suggesting that rtPA-Fuco-SPs do not provoke acute toxicity under the current conditions.

Cerebral blood flow was monitored throughout the treatment via laser Doppler speckle contrast imaging, a high resolution and high-speed technique that instantly visualizes microcirculatory tissue blood perfusion. The reduction of the blood flow in the ipsilateral cerebral hemisphere due to the stroke was restored by $33.97 \pm 5.15 \%$ after 40 min treatment 
with rtPA-Fuco-SPs; by contrast, in the rtPA and saline group for which the perfusion was improved only by $15.16 \pm 6.49 \%$ and $10.33 \pm 4.62 \%$, respectively, in this experiment (Figure 6D). The representative laser Doppler speckle multispectral imaging in the ipsilateral and the contralateral hemispheres at $0 \mathrm{~min}$ and $40 \mathrm{~min}$ are expressed in Figure 6C. Accelerated Video S1A, B, C recorded the perfusion in real-time for all conditions. These data were confirmed by the angiographic analysis performed 24 hours later and assessed by a blinded observer based on TICI grade flow scoring measured by magnetic resonance angiography (MRA). Indeed, similar to some untreated stroke patients, the blood clots were gradually lysed post-stroke in this murine model: at $24 \mathrm{~h}$ after thrombotic occlusion, $40 \%$ of mice exhibited a total recanalization (score 3) and $60 \%$ partial perfusion (score 1 and score 2) of the MCA when injected with saline (Figure 6G; Figure S5A, Supplementary Data). However, after the treatment with rtPA-Fuco-SPs, most of the cases were entirely recanalized (score 3). For the rtPA treated group, no animal achieved a score 1 of minimal perfusion, and $66.6 \%$ of cases showed a partial score 2 level of recanalization.

We then utilized magnetic resonance imaging as a powerful technique to quantify the volume of brain damage at 24 hours after stroke onset. Using T2-weighted MRI sequences, we observed that the size of the ischemic brain lesions for the control saline group was $14.80 \pm$ $3.34 \mathrm{~mm}^{3}$ whereas for the rtPA-loaded fucoidan-targeted SPs it was $4.63 \pm 1.59 \mathrm{~mm}^{3}$ (Figure $6 \mathrm{E} \& 6 \mathrm{~F})$. rtPA-conjugated Fuco-SPs reduced the ischemic zone almost 2-fold of the free rtPA, despite the fact that these data were non-significant due to the limited number of the animals as to the well-known effect of free rtPA in this murine model.[50] Out of all treated animals with rtPA-Fuco-SPs, $66.6 \%$ of the animals displayed small lesion sizes $\left(<3 \mathrm{~mm}^{3}\right)$, and $33.3 \%$ had medium lesion sizes $\left(<11 \mathrm{~mm}^{3}\right)$. In the rtPA group, only $33.3 \%$ of the animals displayed a small infarct zone. In the saline treatment group, we recorded $60 \%$ of the animals with severe $(<20$ 
$\mathrm{mm}^{3}$ ) and critical $\left(>20 \mathrm{~mm}^{3}\right)$ lesions and no animals with small lesions (Figure S5B,

Supplementary Data).

To monitor the BBB integrity disrupted by ischemic stroke,[52] extravasation of gadolinium injected intravenously was detected and quantified on T1-weighted MRI images at day 4 after stroke (Figure $6 \mathrm{H} \& \mathbf{6 I}$ ). Gadolinium extravasation from the blood into the brain parenchyma was unmistakably located at the ischemia-affected region where the BBB is compromised. rtPA-Fuco-SPs treatment group demonstrated a significant BBB preservation over control groups of free rtPA and saline with only a subtle BBB breakdown of $3.45 \pm 1.40$ $\mathrm{mm}^{3}$.

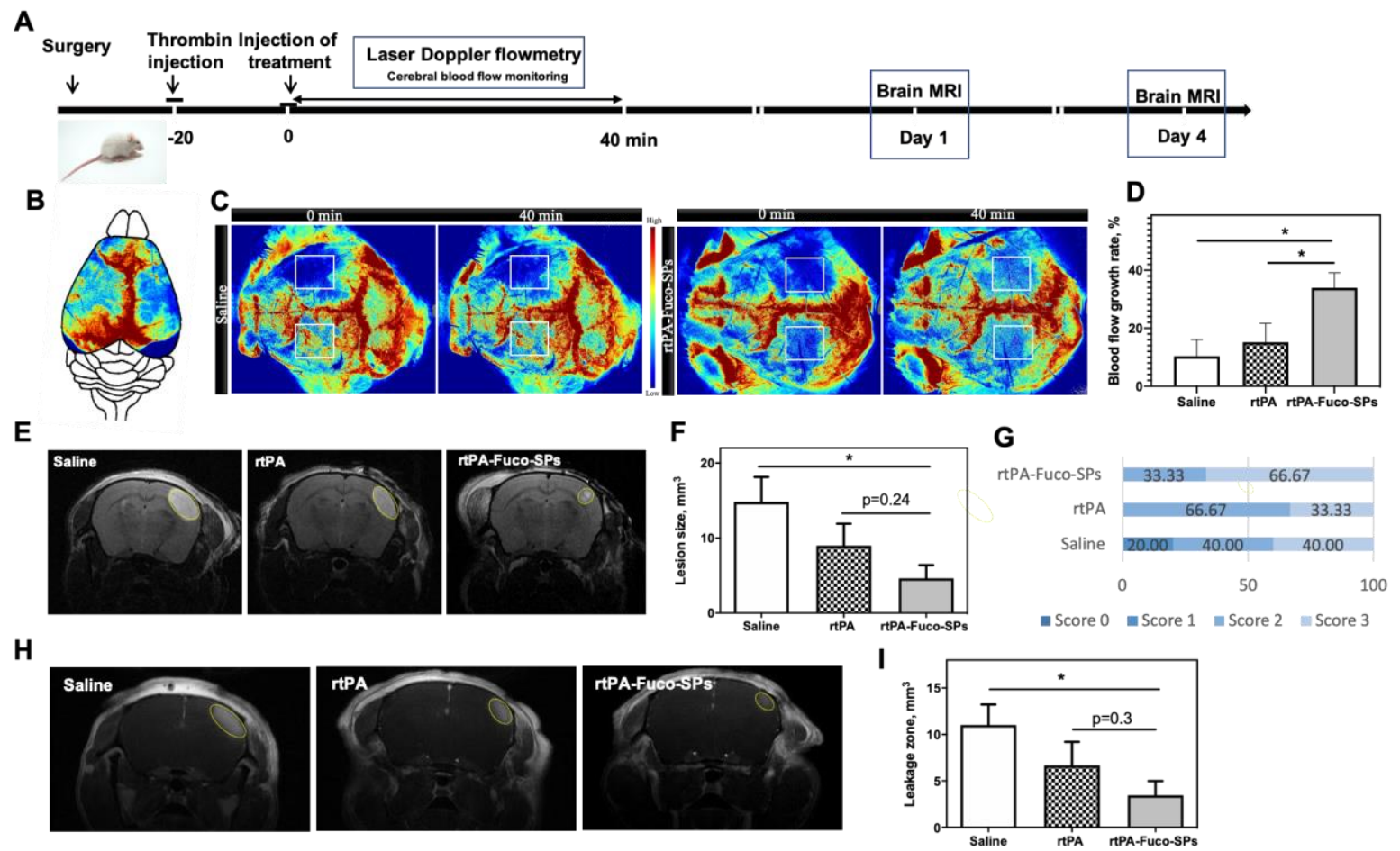

Figure 6. Thrombolytic efficacy in vivo in the murine ischemic stroke model. A. Schematic of the design of the in vivo experiment. B - D. Cerebral blood flow reperfusion monitored by the laser speckle contrast imaging during the 40-min treatment ( $\mathrm{n} \geq 5$ ). Schematic image of the perfusion in the microcirculatory brain tissue. (B). The representative multispectral photos in the ROI of ipsilateral (upper) and contralateral (lower) hemispheres at 0 min and $40 \mathrm{~min}$ for saline and rtPA-Fuco-SPs groups $(\mathbf{C})$. The growth rate of the cerebral reperfusion in the ipsilateral hemisphere after $40 \mathrm{~min}$ treatment injection (D). E. MRI images of the infarct lesion $24 \mathrm{~h}$ post-stroke with the corresponding quantification of its volume $(\mathbf{F}),(\mathrm{n} \geq 5)$. $\mathbf{G}$. Angiographic scores of the MCA reperfusion at day 1 after stroke induction by MRI. H. MRI images of the BBB permeability at day 4 post-stroke with the corresponding quantification of its volume $(\mathbf{I}),(\mathrm{n} \geq 5)$. 
In order to confirm the accumulation of Fuco-SPs within the thrombus area in vivo, we injected fluorescent-labeled SPs at the therapeutic concentration as in the stroke model into the $\mathrm{FeCl}_{3}$ model of venous thrombosis in mice. Upon histological examination of the thrombusaffected area of the mesenteric vein, Fuco-SPs were clearly identified on the green-fluorescent labeled thrombus (Figure 7A). By a quantitative analysis of the MFI normalized by the size of the thrombus, it was evidenced a highly increased TRITC signal from fucoidan-functionalized SPs group comparing with Control-SPs $(9.48 \pm 2.19$ for Fuco-SPs vs. $1.48 \pm 0.35$ for ControlSPs, ${ }^{* *} \mathrm{p}<0.01$, Figure 7B).
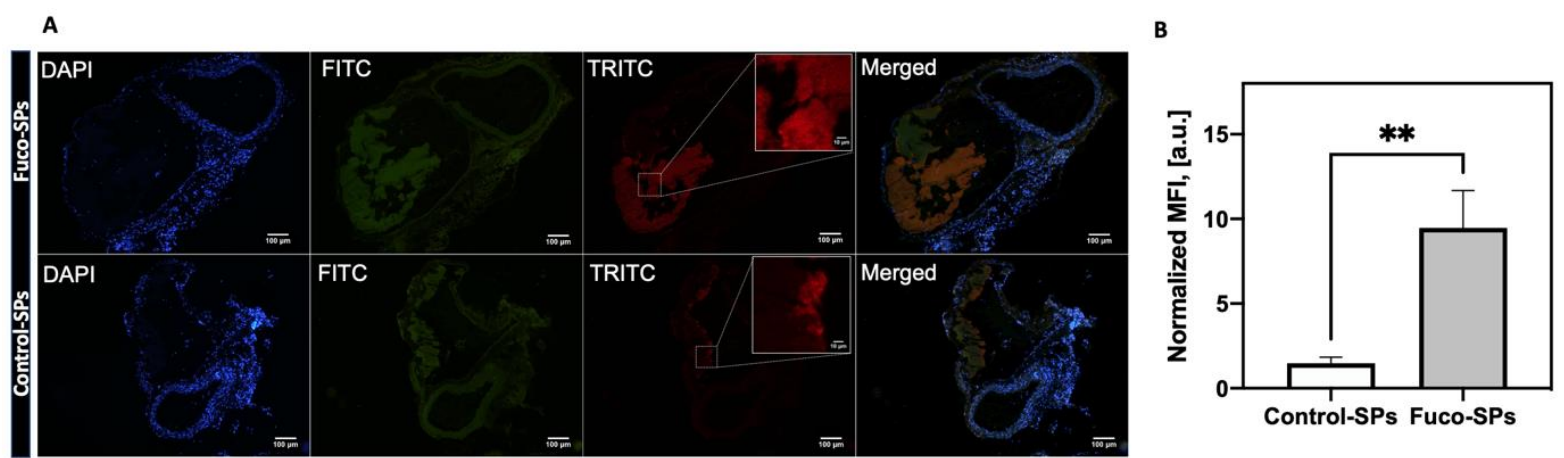

Figure 7. In vivo targeting in the $\mathrm{FeCl}_{3}$ murine model of thrombosis. A. Histological analysis of thrombi in the mesenteric vein by fluorescent microscopy. The venous vascular wall is visualized with DAPI staining; thrombus is stained in green due to DIOC6 labeling of platelets; Fuco-SPs and Control-SPs are fluorescently labeled with TRITC. Inserts are a magnification of dashed Regions of Interest of the TRITC signal. B. Corresponding quantification of MFI from the adhered TRITC-SPs in the thrombi $(n=5)$.

Overall, the apparent superiority of rtPA-Fuco-SPs to reduce the brain tissue injury area in comparison with saline and rtPA at the same dose, combined with a favorable safety profile of the SPs, makes them a promising nanomedicine-based approach for the treatment of acute arterial thrombosis.

\section{DISCUSSION}

The revolutionary treatment with alteplase to dissolve thrombi and restore blood flow is a standard of care in acute thrombotic events, but it is certainly not risk-free. Nanomedicine exploits a profound understanding of the molecular mechanisms involved in thrombus 
formation and offers outside-the-box strategies to effectively target and dissolve the blood clots in the body with fewer systemic and neurological complications.[53]

In this study, rtPA was associated with hydrogel-like polysaccharide SPs, synthesized by a simple and reproducible miniemulsion / crosslinking process. The soft structure of chemically crosslinked hydrogels resembles those of extracellular matrices and may prevent tissue irritation, providing a convenient and versatile platform for storage and site-specific delivery of a drug to the thrombi.[54] Our approach's novelty is exclusively polysaccharide nature of the particles - from dextran and fucoidan without any chemical modification, as well as green chemistry synthesis technique. Intriguingly, several articles claim that dextran[55] and fucoidan[56,57] themselves exert some antithrombotic action that makes them an excellent starting material for the nanocarrier. Hydrophilic polysaccharide surfaces lead to longer circulating NPs since they avert the formation of a protein corona and early macrophage internalization.[58] Moreover, natural polysaccharides are advantageous to synthetic polymers in terms of safety, abundance in nature, and low cost. Overall, we considered the issues imperative for high biosafety and potential future clinical translation of Fuco-SPs by (I) the choice of biocompatible and biodegradable materials, all with FDA-approval; (II) a relatively simple and efficient production by a mild synthesis method without the utilization of the hazardous substances and organic solvents; (III) targeting agent of natural origin - fucoidan that is being validated in clinical trials for thrombosis.

After fabrication and extensive physico-chemical characterization of the SPs, we confirmed their cyto- and hemocompatibility at concentrations up to $1.5 \mathrm{mg} / \mathrm{ml}$ (Figure $2 \mathrm{~A} \&$ 3B). The main pathway of the polysaccharide Fuco-SPs tissue distribution in vivo upon intravenous injection was the spleen (Figure S4, Supplementary Data). Future studies should include the tests of thrombogenicity and immunogenicity in vivo. 
The Fuco-SPs were associated with a thrombolytic agent by physical adsorption, allowing a high encapsulation efficiency of the rtPA of $81.04 \pm 1.86 \%$ due to their hydrogelbased structure. Classically, alteplase is loaded onto the NPs using the covalent amide bond formation via EDC/NHS reaction between the primary amines on the rtPA molecule and the nanocarriers containing carboxyl groups. As a mild drug encapsulation method, adsorption is preferred to covalent bioconjugation to load fragile molecules such as proteins to avoid changes in the protein structure and function that might result in its partial denaturation and loss of biological activity.[59] The fibrinolytic diffused through the matrix of the porous SPs (Figure S2A, Supplementary Data), fully preserving its enzymatic and fibrinolytic potential in vitro (Figure 4). Friedrich et al. demonstrated that the adsorptive bound rtPA liberated faster from the particles and spread more readily into the fibrin matrix than covalently bound rtPA,[60] which may be beneficial in targeted thrombolysis. Although a rising number of recent articles apply a controlled release of the thrombolytics via endogenous and exogenous stimuli, we chose not to incorporate sophisticated nanodesigns and demonstrated a gradual and continuous rtPA release from the SPs without an external trigger (Figure S2B, Supplementary Data) so as to facilitate future regulatory approvals.

The clot targeting strategy of the SPs was achieved by the introduction of the fucoidan to the polysaccharide solution at $9 \%$ w/w. Functionalization with fucoidan permitted the highaffinity binding of Fuco-SPs to its molecular target P-selectin in a dynamic microfluidic method that mimics arterial or venous blood flow conditions in a dose-dependent manner (Figure 3AB, AE). Importantly, this type of interaction is selective, as SPs did not adhere to other selectins: E- and L-selectin in vitro (Figure 3D). When the microchannels were covered with activated platelet aggregates, both unloaded and rtPA-loaded Fuco-SPs were capable of binding these micro-clots (Figure 5). Once tested in the in vivo model of venous thrombosis, Fuco-SPs were identified on the $\mathrm{FeCl}_{3}$-induced thrombi by histological examination, contrary to Control-SPs 
(Figure 7). Our data validate an inimitable and efficient thrombus targeting approach of the

Fuco-SPs, using a clinical algae-derived compound. Yet, a surge in strategies involving dual or even more surface targeting moieties is being witnessed in academic works. For instance, the combinatory targeting of GPIIb/IIIa receptors and P-selectin on activated platelets with two peptide ligands on the lipid nanovesicles enhanced thrombus binding capacities in a synergic manner.[61] Another notable example is the biomimetic functionalization of NPs with platelet membrane coating that enabled thrombus directing via multiple platelet receptors.[62] These combinations of NP modifications represent a promising strategy for developing nextgeneration blood clot-targeting NP platforms for therapy and/or diagnostics.

The experiments on the murine thrombin-induced thromboembolic stroke model supported the therapeutic effect of the rtPA-Fuco-SPs in vivo (Figure 6). The findings of the submicroparticle-associated rtPA treatment group outperformed those of free rtPA at the same concentration, as we observed a more rapid clot dissolution and MCA reperfusion, which minimized the brain injury and resulted in subsequently smaller infarct area post-stroke by MRI. The prevailing method for assessing the brain infarct volume is a brain tissue staining with 2\% 2,3,5-triphenyltetrazolium chloride (TTC), which labels non-injured tissue and leaves the infarct area white. Several groups previously reported the reduction of the infarct zone in preclinical models by nanomedicinal product. For example, the magnetic iron oxide $\left(\mathrm{Fe}_{3} \mathrm{O}_{4}\right)$ microrods[63] and polyacrylic acid-stabilized magnetic NPs[64] conjugated with rtPA diminished the brain infarct lesion in the $\mathrm{FeCl}_{3}$ murine model of ischemic stroke of MCA. These designs, however, require an external magnet for targeting and to complement chemical lysis with rtPA with mechanical one of the magnetic rotations. Without any clinically approved medical device to impose a high magnetic force on the NPs in deep blood vessels, it would probably be desirable for nanocarrier formulation to avoid external assistance. Mei et al. stated that only the synergistic effect of rtPA-loaded polymer micelles and a reactive oxygen species 
(ROS)-eliminating antioxidant suppressed an infarct volume and improved neurological deficit after brain ischemia in the mouse model of photo-thrombotic MCA occlusion.[65] In our case, the therapeutic benefit of rtPA-Fuco-SPs could be ascribed to faster MCA reperfusion and, hence, prevention of the major brain injury due to higher rtPA accumulation on the thrombus site as to active targeting and specific P-selectin interactions of the Fuco-SPs.

Moreover, the animals in the rtPA-Fuco-SPs group suffered a lower level of BBB disruption by the ischemic stroke that was quantified on T1-weighted MRI images with gadolinium. It is vital to underline that although in this article we utilized fucoidan on the SPs purely for the P-selectin targeting purpose, new avenues of research are deciphering its neuroprotective role, particularly after cerebral ischemic events.[66] Altogether, this first proofof-concept in vivo results of Fuco-SPs show an astonishing potential of the nanomedicine-based approach for the targeted treatment of acute thrombosis. We speculate that the submicron size of the particles, along with their active thrombus-targeting moiety, should maintain rtPA-loaded nanocarrier within the intravascular compartment to exert its thrombolytic activity. It should prevent the leakage of rtPA into the brain parenchyma, reducing the risks of NMDA receptorsmediated neurotoxicity and hemorrhages.[67] Further studies could compare a single or a double bolus route of administration of rtPA-Fuco-SPs due to the rtPA preservation by SPs, and, thus, a more comfortable treatment option for patients.

Nevertheless, as none of the available in vivo rodent experimental models of acute thrombosis accurately recapitulates all the aspects of human disease progression and heterogeneity, it is recommended to validate any translational concept in multiple models to ensure its therapeutic efficacy and safety. Notably, in 2019, Xu et al. proposed a biomimetic strategy of platelet membrane-camouflaged PLGA NPs with rtPA that was studied in several in vivo models: pulmonary embolism, $\mathrm{FeCl}_{3}$-induced arterial thrombosis, and ischemic stroke model.[62] 
Due to technical challenges and strict regulatory requirements, the clinical translation of the nanopharmaceutical products happens slowly. The rtPA-Fuco-SPs representing an actively targeted nanodelivery system reach a balance of safety, simplicity, and functionality concerning the projected clinical application. While they were designed and fabricated in the academic laboratory at the range of $\sim 10 \mathrm{mg}$ per batch, the industrial scale-up of the process with high reproducibility under GMP conditions and intended quality standards is essential to establish in order to obtain the necessary amounts for further screening and, ultimately, clinical use. For instance, high-precision microfluidic and micropatterning methods for the fabrication of monodisperse NPs with uniform physicochemical characteristics and low batch-to-batch variability are becoming prevalent and are compatible with GMP standards.[68] Finally, an adequate sterilization method should be validated to prevent nanoparticle damage and alteration in the product's parameters.

\section{CONCLUSIONS}

In the present study, we designed and fabricated fucoidan-functionalized $100 \%$ polysaccharide submicroparticles from biocompatible and FDA approved components as a P-selectin targeting drug delivery system for thrombolytic therapy. The physico-chemical properties and a biocompatibility analysis of these SPs were thoroughly evaluated, and alteplase was effectively immobilized onto the SPs with full retention of its enzymatic and fibrinolytic potential in vitro and sustained drug-release kinetics. We established in vitro by dynamic flow microchamber assays that the fucoidan-functionalized nanosystem specifically adhered to the recombinant Pselectin in a dose-dependent manner, but not to E- and L-Selectins, and to human activated platelets. In $\mathrm{FeCl}_{3}$ model of thrombosis, Fuco-SPs accumulated in the thrombus. Finally, our findings revealed in the murine model of ischemic stroke that rtPA conjugation to the Fuco-SPs could enhance the thrombolytic activity of the clinical agent in vivo. The blood flow perfusion 
was restored more rapidly, which resulted in smaller post-ischemic cerebral infarct lesions and higher BBB protection. In summary, an alteplase-associated hydrogel-based nano-delivery system with fucoidan demonstrates a solid argument for improved thrombolytic therapy in terms of safety and efficacy in the preclinical studies. In future research, our biocompatible Fuco-SPs could also vehicle other therapeutic and/or molecular imaging agents in the vascular compartment to target P-selectin overexpressed pathologies, such as cardiovascular diseases[69] or some cancers.[70,71]

\section{Acknowledgments}

The authors would like to acknowledge Marie-Françoise Bricot from the Institut de Chimie des Substances Naturelles of the Université Paris-Saclay for performing a microanalysis of the fucoidan and the Fuco-SPs. We also thank the ImagoSeine core facility of the Institut Jacques Monod, a member of IBiSA and France-Bio-Imaging (ANR-10-INBS-04) infrastructures for the TEM. The authors are grateful for Christine Choqueux, who performed the Environmental SEM at Université de Technologie de Compiègne, Compiègne, France. We kindly thank Pr. Antonino Nicoletti for his advice on designing an in vitro release study and Dr. Kevin Guedj for assistance with flow cytometry. We also thank Dr. Yoann Lalatonne for the technical support with the TXRF technique and Dr. Samira Benadda with confocal microscopy. The illustrations were designed with BioRender software (biorender.com). Funding: The authors thank INSERM, Université de Paris, and Université Sorbonne Paris Nord for financial support. This work also received funding from the EU project FP7-NMP-2012-LARGE-6-309820 "NanoAthero" and ANR-13-LAB1-0005-01 "FucoChem". Alina Zenych is grateful for the $\mathrm{Ph} . \mathrm{D}$. fellowship from the INSPIRE program of the European Union's Horizon 2020 research and innovation program (Marie Sklodowska-Curie grant \# 665850).

\section{Author contributions}

A.Z.: Conceptualization; Investigation; Data Curation; Formal analysis; Writing - Original Draft; Visualization. C.J.: Investigation (Thrombolytic efficacy in vivo). R.A.: Investigation (Tissue distribution in vivo \& thrombus targeting in vivo); Validation. L.F.: Investigation (Quantification of the fucoidan \& fibrinolytic activity in vitro). L.M.F.R.: Methodology. T.B.: Methodology; Investigation (Thrombolytic efficacy in vivo - MRI); Validation; Writing Review \& Editing. D.V.: Resources; Project administration; Writing - Review \& Editing. D.L.: Resources; Project administration. C.C.: Conceptualization; Validation; Resources; Writing Review \& Editing; Supervision; Project administration; Funding acquisition. All the authors approved the final version of the manuscript.

\section{Conflict of interests}

The authors declare no competing interests. 


\section{REFERENCES}

[1] E.J. Benjamin, P. Muntner, A. Alonso, M.S. Bittencourt, C.W. Callaway, A.P. Carson, A.M. Chamberlain, A.R. Chang, S. Cheng, S.R. Das, F.N. Delling, L. Djousse, M.S.V. Elkind, J.F. Ferguson, M. Fornage, L.C. Jordan, S.S. Khan, B.M. Kissela, K.L. Knutson, T.W. Kwan, D.T. Lackland, T.T. Lewis, J.H. Lichtman, C.T. Longenecker, M.S. Loop, P.L. Lutsey, S.S. Martin, K. Matsushita, A.E. Moran, M.E. Mussolino, M. O’Flaherty, A. Pandey, A.M. Perak, W.D. Rosamond, G.A. Roth, U.K.A. Sampson, G.M. Satou, E.B. Schroeder, S.H. Shah, N.L. Spartano, A. Stokes, D.L. Tirschwell, C.W. Tsao, M.P. Turakhia, L.B. VanWagner, J.T. Wilkins, S.S. Wong, S.S. Virani, Heart Disease and Stroke Statistics-2019 Update: A Report From the American Heart Association, Circulation. (2019). doi:10.1161/CIR.0000000000000659.

[2] W.J. Powers, A.A. Rabinstein, T. Ackerson, O.M. Adeoye, N.C. Bambakidis, K. Becker, J. Biller, M. Brown, B.M. Demaerschalk, B. Hoh, E.C. Jauch, C.S. Kidwell, T.M. LeslieMazwi, B. Ovbiagele, P.A. Scott, K.N. Sheth, A.M. Southerland, D. V. Summers, D.L. Tirschwell, Guidelines for the Early Management of Patients With Acute Ischemic Stroke: 2019 Update to the 2018 Guidelines for the Early Management of Acute Ischemic Stroke: A Guideline for Healthcare Professionals From the American Heart Association/American Stroke , Stroke. $50 \quad$ (2019) e344-e418. doi:10.1161/STR.0000000000000211.

[3] J. Mican, M. Toul, D. Bednar, J. Damborsky, Structural Biology and Protein Engineering of Thrombolytics, Comput. Struct. Biotechnol. J. 17 (2019) 917-938. doi:10.1016/j.csbj.2019.06.023.

[4] R. Bhatia, M.D. Hill, N. Shobha, B. Menon, S. Bal, P. Kochar, T. Watson, M. Goyal, A.M. Demchuk, Low rates of acute recanalization with intravenous recombinant tissue plasminogen activator in ischemic stroke: Real-world experience and a call for action, Stroke. 41 (2010) 2254-2258. doi:10.1161/STROKEAHA.110.592535.

[5] A.M. Thiebaut, M. Gauberti, C. Ali, S. Martinez De Lizarrondo, D. Vivien, M. Yepes, B.D. Roussel, The role of plasminogen activators in stroke treatment: fibrinolysis and beyond, Lancet Neurol. 17 (2018) 1121-1132. doi:10.1016/S1474-4422(18)30323-5.

[6] A. Pal Khasa, Y. Pal Khasa, The evolution of recombinant thrombolytics: Current status and future directions, Bioengineered. $8 \quad$ (2017) 331-358. doi:10.1080/21655979.2016.1229718.

[7] S. Liu, X. Feng, R. Jin, G. Li, Tissue plasminogen activator-based nanothrombolysis for ischemic stroke, Expert Opin Drug Deliv. 15 (2018) 173-184. doi:10.1080/17425247.2018.1384464.

[8] N. Korin, M. Kanapathipillai, B.D. Matthews, M. Crescente, A. Brill, T. Mammoto, K. Ghosh, S. Jurek, S.A. Bencherif, D. Bhatta, A.U. Coskun, C.L. Feldman, D.D. Wagner, D.E. Ingber, Shear-Activated Nanotherapeutics for Drug Targeting to Obstructed Blood Vessels, Science (80-. ). 337 (2012) 738-742. doi:10.1126/science.1217815.

[9] M. Colasuonno, A.L. Palange, R. Aid, M. Ferreira, H. Mollica, R. Palomba, M. Emdin, M. Del Sette, C. Chauvierre, D. Letourneur, P. Decuzzi, Erythrocyte-Inspired Discoidal Polymeric Nanoconstructs Carrying Tissue Plasminogen Activator for the Enhanced Lysis of Blood Clots, ACS Nano. 12 (2018) 12224-12237. doi:10.1021/acsnano.8b06021.

[10] J. Zhou, D. Guo, Y. Zhang, W. Wu, H. Ran, Z. Wang, Construction and evaluation of Fe3O4-based PLGA nanoparticles carrying rtPA used in the detection of thrombosis and in targeted thrombolysis, ACS Appl. Mater. Interfaces. 6 (2014) 5566-5576. doi:10.1021/am406008k.

[11] A. Friedman, S. Claypool, R. Liu, The Smart Targeting of Nanoparticles, Curr. Pharm. 
Des. 19 (2013) 6315-6329. doi:10.2174/13816128113199990375.

[12] W. jin Jeong, J. Bu, L.J. Kubiatowicz, S.S. Chen, Y.S. Kim, S. Hong, Peptidenanoparticle conjugates: a next generation of diagnostic and therapeutic platforms?, Nano Converg. 5 (2018) 1-18. doi:10.1186/s40580-018-0170-1.

[13] L. Chollet, P. Saboural, C. Chauvierre, J.-N. Villemin, D. Letourneur, F. Chaubet, Fucoidans in Nanomedicine, Mar. Drugs. 14 (2016) 1-24. doi:10.3390/md14080145.

[14] L. Bachelet, I. Bertholon, D. Lavigne, R. Vassy, M. Jandrot-Perrus, F. Chaubet, D. Letourneur, Affinity of low molecular weight fucoidan for P-selectin triggers its binding to activated human platelets, Biochim. Biophys. Acta - Gen. Subj. 1790 (2009) 141-146. doi:10.1016/j.bbagen.2008.10.008.

[15] P. Saboural, F. Chaubet, F. Rouzet, F. Al-Shoukr, R. Ben Azzouna, N. Bouchemal, L. Picton, L. Louedec, M. Maire, L. Rolland, G. Potier, D. Le Guludec, D. Letourneur, C. Chauvierre, Purification of a low molecular weight fucoidan for SPECT molecular imaging of myocardial infarction, Mar. Drugs. 12 (2014) 4851-4867. doi: $10.3390 / \mathrm{md} 12094851$.

[16] T. Bonnard, J.-M. Serfaty, C. Journé, B. Ho Tin Noe, D. Arnaud, L. Louedec, M. Derkaoui, D. Letourneur, C. Chauvierre, C. Le Visage, Leukocyte mimetic polysaccharide microparticles tracked in vivo on activated endothelium and in abdominal aortic aneurysm, Acta Biomater. 10 (2014) 3535-3545. doi:10.1016/j.actbio.2014.04.015.

[17] T. Bonnard, G. Yang, A. Petiet, V. Ollivier, O. Haddad, D. Arnaud, L. Louedec, L. Bachelet-Violette, S.M. Derkaoui, D. Letourneur, C. Chauvierre, C. Le Visage, Abdominal Aortic Aneurysms Targeted by Functionalized Polysaccharide Microparticles: a new Tool for SPECT Imaging, Theranostics. 4 (2014) 592-603. doi:10.7150/thno.7757.

[18] B. Li, M. Juenet, R. Aid-Launais, M. Maire, V. Ollivier, D. Letourneur, C. Chauvierre, Development of Polymer Microcapsules Functionalized with Fucoidan to Target PSelectin Overexpressed in Cardiovascular Diseases, Adv. Healthc. Mater. 6 (2017) 111. doi:10.1002/adhm.201601200.

[19] B. Li, R. Aid-Launais, M.-N. Labour, A. Zenych, M. Juenet, C. Choqueux, V. Ollivier, O. Couture, D. Letourneur, C. Chauvierre, Functionalized polymer microbubbles as new molecular ultrasound contrast agent to target P-selectin in thrombus, Biomaterials. 194 (2019) 139-150. doi:10.1016/j.biomaterials.2018.12.023.

[20] M. Juenet, R. Aid-Launais, B. Li, A. Berger, J. Aerts, V. Ollivier, A. Nicoletti, D. Letourneur, C. Chauvierre, Thrombolytic therapy based on fucoidan-functionalized polymer nanoparticles targeting P-selectin, Biomaterials. 156 (2018) 204-216. doi:10.1016/j.biomaterials.2017.11.047.

[21] C. Chauvierre, D. Letourneur, The European project NanoAthero to fight cardiovascular diseases using nanotechnologies, Nanomedicine (Lond). 10 (2015) 3391-3400. doi:10.2217/nnm.15.170.

[22] K.H. Zheng, Y. Kaiser, E. Poel, H. Verberne, J. Aerts, F. Rouzet, E. Stroes, D. Letourneur, C. Chauvierre, 99Mtc-Fucoidan As Diagnostic Agent For P-Selectin Imaging: First-In-Human Evaluation (Phase I), Atherosclerosis. 287 (2019) e143. doi:10.1016/j.atherosclerosis.2019.06.425.

[23] I. Cicha, C. Chauvierre, I. Texier, C. Cabella, J.M. Metselaar, J. Szebeni, L. Dézsi, C. Alexiou, F. Rouzet, G. Storm, E. Stroes, D. Bruce, N. MacRitchie, P. Maffia, D. Letourneur, From design to the clinic: Practical guidelines for translating cardiovascular nanomedicine, Cardiovasc. Res. 114 (2018) 1714-1727. doi:10.1093/cvr/cvy219.

[24] K. Ganguly, K. Chaturvedi, U.A. More, M.N. Nadagouda, T.M. Aminabhavi, Polysaccharide-based micro/nanohydrogels for delivering macromolecular therapeutics, 
J. Control. Release. 193 (2014) 162-173. doi:10.1016/j.jconrel.2014.05.014.

[25] J. Zhang, W. Xia, P. Liu, Q. Cheng, T. Tahirou, W. Gu, B. Li, Chitosan modification and pharmaceutical/biomedical applications, Mar. Drugs. 8 (2010) 1962-1987. doi:10.3390/md8071962.

[26] H.J. Jin, H. Zhang, M.L. Sun, B.G. Zhang, J.W. Zhang, Urokinase-coated chitosan nanoparticles for thrombolytic therapy: Preparation and pharmacodynamics in vivo, J. Thromb. Thrombolysis. 36 (2013) 458-468. doi:10.1007/s11239-013-0951-7.

[27] J. Liao, X. Ren, B. Yang, H. Li, Y. Zhang, Z. Yin, Targeted thrombolysis by using cRGD-modified N,N,N-Trimethyl Chitosan nanoparticles loaded with lumbrokinase, Drug Dev. Ind. Pharm. 45 (2019) 88-95. doi:10.1080/03639045.2018.1522324.

[28] S.R. Van Tomme, W.E. Hennink, Biodegradable dextran hydrogels for protein delivery applications, Expert Rev. Med. Devices. 4 (2007) 147-164. doi:10.1586/17434440.4.2.147.

[29] J.R. McCarthy, I.Y. Sazonova, S.S. Erdem, T. Hara, B.D. Thompson, P. Patel, I. Botnaru, C.P. Lin, G.L. Reed, R. Weissleder, F.A. Jaffer, Multifunctional nanoagent for thrombus-targeted fibrinolytic therapy, Nanomedicine (Lond). 7 (2012) 1017-1028. doi:10.2217/nnm.11.179.

[30] S. Heid, H. Unterweger, R. Tietze, R.P. Friedrich, B. Weigel, I. Cicha, D. Eberbeck, A.R. Boccaccini, C. Alexiou, S. Lyer, Synthesis and Characterization of Tissue Plasminogen Activator-Functionalized Superparamagnetic Iron Oxide Nanoparticles for Targeted Fibrin Clot Dissolution, Int. J. Mol. Sci. 18 (2017). doi:10.3390/ijms18091837.

[31] T.E. Furia, CRC Handbook of Food Additives, Second Edi, CRC Press, 1973.

[32] W.E. Hennink, C.F. van Nostrum, Novel crosslinking methods to design hydrogels, Adv. Drug Deliv. Rev. 54 (2002) 13-36. doi:10.1016/S0169-409X(01)00240-X.

[33] C. Chauvierre, R. Aid-Launais, J. Aerts, M. Maire, L. Chollet, L. Rolland, R. Bonaf, S. Rossi, S. Bussi, C. Cabella, D. Laszlo, T. Fülöp, J. Szebeni, Y. Chahid, K.H. Zheng, E.S.G. Stroes, D. Le Guludec, F. Rouzet, D. Letourneur, Pharmaceutical Development and Safety Evaluation of a GMP-Grade Fucoidan for Molecular Diagnosis of Cardiovascular Diseases, Mar. Drugs. 17 (2019) 1-17. doi:https://doi.org/10.3390/md17120699.

[34] F. Rouzet, L. Bachelet-Violette, J.-M. Alsac, M. Suzuki, A. Meulemans, L. Louedec, A. Petiet, M. Jandrot-Perrus, F. Chaubet, J.-B. Michel, D. Le Guludec, D. Letourneur, Radiolabeled fucoidan as a P-selectin targeting agent for in vivo imaging of platelet-rich thrombus and endothelial activation., J. Nucl. Med. 52 (2011) 1433-1440. doi:10.2967/jnumed.110.085852.

[35] F. Wolf, K. Koehler, H.P. Schuchmann, Stabilization of water droplets in oil with PGPR for use in oral and dermal applications, J. Food Process Eng. 36 (2013) 276-283. doi:10.1111/j.1745-4530.2012.00688.x.

[36] J. Matuszak, J. Baumgartner, J. Zaloga, M. Juenet, A.E. da Silva, D. Franke, G. Almer, I. Texier, D. Faivre, J.M. Metselaar, F.P. Navarro, C. Chauvierre, R. Prassl, L. Dézsi, R. Urbanics, C. Alexiou, H. Mangge, J. Szebeni, D. Letourneur, I. Cicha, Nanoparticles for intravascular applications: physicochemical characterization and cytotoxicity testing, Nanomedicine. 11 (2016) 597-616. doi:10.2217/nnm.15.216.

[37] M. Weber, H. Steinle, S. Golombek, L. Hann, C. Schlensak, H.P. Wendel, M. AvciAdali, Blood-Contacting Biomaterials: In Vitro Evaluation of the Hemocompatibility, Front. Bioeng. Biotechnol. 6 (2018) 99. doi:10.3389/fbioe.2018.00099.

[38] ISO 10993-4:2017, Biol. Eval. Med. Devices — Part 4 Sel. Tests Interact. with Blood. (2017). https://www.iso.org/standard/63448.html.

[39] B. Vaidya, G.P. Agrawal, S.P. Vyas, Platelets directed liposomes for the delivery of streptokinase: Development and characterization, Eur. J. Pharm. Sci. 44 (2011) 589-594. 
doi:10.1016/j.ejps.2011.10.004.

[40] N. Zhang, C. Li, D. Zhou, C. Ding, Y. Jin, Q. Tian, X. Meng, K. Pu, Y. Zhu, Cyclic RGD functionalized liposomes encapsulating urokinase for thrombolysis, Acta Biomater. (2018). doi:10.1016/j.actbio.2018.01.038.

[41] K. Ley, The role of selectins in inflammation and disease, Trends Mol. Med. 9 (2003) 263-268. doi:10.1016/S1471-4914(03)00071-6.

[42] J.-H. Kim, J.-Y. Yoon, Protein adsorption on polymer particles, in: Encycl. Surf. Colloid Sci., 2002: pp. 4373-4381. doi:10.1002/jbm.820210202.

[43] I. Politis, L. Wang, J.D. Turner, B.K. Tsang, Changes in Tissue-Type Plasminogen Activator-Like and Plasminogen Activator Inhibitor Activities in Granulosa and Theca Layers during Ovarian Follicle Development in the Domestic Hen1, Biol. Reprod. 42 (1990) 747-754. doi:10.1095/biolreprod42.5.747.

[44] S. Petersen, A. Fahr, H. Bunjes, Flow cytometry as a new approach to investigate drug transfer between lipid particles, Mol. Pharm. 7 (2010) 350-363. doi:10.1021/mp900130s.

[45] V. Wintgens, C. Lorthioir, P. Dubot, B. Sébille, C. Amiel, Cyclodextrin/dextran based hydrogels prepared by cross-linking with sodium trimetaphosphate, Carbohydr. Polym. 132 (2015) 80-88. doi:10.1016/j.carbpol.2015.06.038.

[46] L. Arnfast, C.G. Madsen, L. Jorgensen, S. Baldursdottir, Design and processing of nanogels as delivery systems for peptides and proteins, Ther. Deliv. 5 (2014) 691-708. doi:10.4155/tde.14.38.

[47] Z.M. Ruggeri, Platelets in atherothrombosis, Nat. Med. 8 (2002) 1227-1234. doi:10.4065/81.1.59.

[48] T.J. Merkel, K. Chen, S.W. Jones, A.A. Pandya, S. Tian, M.E. Napier, W.E. Zamboni, J.M. Desimone, The effect of particle size on the biodistribution of low-modulus hydrogel PRINT particles, J. Control. Release. 162 (2012) 37-44. doi:10.1016/j.jconrel.2012.06.009.

[49] C. Orset, B. Haelewyn, S.M. Allan, S. Ansar, F. Campos, T.H. Cho, A. Durand, M. El Amki, M. Fatar, I. Garcia-Yébenes, M. Gauberti, S. Grudzenski, I. Lizasoain, E. Lo, R. Macrez, I. Margaill, S. Maysami, S. Meairs, N. Nighoghossian, J. Orbe, J.A. Paramo, J.J. Parienti, N.J. Rothwell, M. Rubio, C. Waeber, A.R. Young, E. Touzé, D. Vivien, Efficacy of Alteplase in a Mouse Model of Acute Ischemic Stroke: A Retrospective Pooled Analysis, Stroke. 47 (2016) 1312-1318. doi:10.1161/STROKEAHA.116.012238.

[50] S.M. De Lizarrondo, C. Gakuba, B.A. Herbig, Y. Repessé, C. Ali, C. V. Denis, P.J. Lenting, E. Touzé, S.L. Diamond, D. Vivien, M. Gauberti, Potent thrombolytic effect of $\mathrm{N}$-acetylcysteine on arterial thrombi, Circulation. 136 (2017) 646-660. doi:10.1161/CIRCULATIONAHA.117.027290.

[51] H.R. Lijnen, B. Van Hoef, V. Beelen, D. Collen, Characterization of the Murine Plasma Fibrinolytic System, Eur. J. Biochem. 224 (1994) 863-871. doi:10.1111/j.14321033.1994.00863.x.

[52] R. Brouns, P.P. De Deyn, The complexity of neurobiological processes in acute ischemic stroke, Clin. Neurol. Neurosurg. 111 (2009) 483-495. doi:10.1016/j.clineuro.2009.04.001.

[53] A. Zenych, L. Fournier, C. Chauvierre, Nanomedicine progress in thrombolytic therapy, Biomaterials. 258 (2020) 120297. doi:10.1016/j.biomaterials.2020.120297.

[54] K.S. Masters, D.N. Shah, L.A. Leinwand, K.S. Anseth, Crosslinked hyaluronan scaffolds as a biologically active carrier for valvular interstitial cells, Biomaterials. 26 (2005) 2517-2525. doi:10.1016/j.biomaterials.2004.07.018.

[55] C.I. Jones, D.A. Payne, P.D. Hayes, A.R. Naylor, P.R.F. Bell, M.M. Thompson, A.H. Goodall, The antithrombotic effect of dextran-40 in man is due to enhanced fibrinolysis in vivo, J. Vasc. Surg. 48 (2008) 715-722. doi:10.1016/j.jvs.2008.04.008. 
[56] E.M. Balboa, E. Conde, A. Moure, E. Falqué, H. Domínguez, In vitro antioxidant properties of crude extracts and compounds from brown algae, Food Chem. 138 (2013) 1764-1785. doi:10.1016/j.foodchem.2012.11.026.

[57] Y. Choi, S.K. Min, R. Usoltseva, A. Silchenko, T. Zvyagintseva, S. Ermakova, J.K. Kim, Thrombolytic fucoidans inhibit the tPA-PAI1 complex, indicating activation of plasma tissue-type plasminogen activator is a mechanism of fucoidan-mediated thrombolysis in a mouse thrombosis model, Thromb. Res. 161 (2018) 22-25. doi:10.1016/j.thromres.2017.11.015.

[58] K. Knop, R. Hoogenboom, D. Fischer, U.S. Schubert, Poly(ethylene glycol) in drug delivery: Pros and cons as well as potential alternatives, Angew. Chemie - Int. Ed. 49 (2010) 6288-6308. doi:10.1002/anie.200902672.

[59] M. Di Marco, K.A. Razak, A.A. Aziz, C. Devaux, E. Borghi, L. Levy, C. Sadun, Overview of the main methods used to combine proteins with nanosystems : absorption, bioconjugation, and encapsulation, Int. J. Nanomedicine. 5 (2010) 37-49.

[60] R.P. Friedrich, J. Zaloga, E. Schreiber, I.Y. Tóth, E. Tombácz, S. Lyer, C. Alexiou, Tissue Plasminogen Activator Binding to Superparamagnetic Iron Oxide NanoparticleCovalent Versus Adsorptive Approach, Nanoscale Res. Lett. 11 (2016) 1-11. doi:10.1186/s11671-016-1521-7.

[61] C.L. Pawlowski, W. Li, M. Sun, K. Ravichandran, D. Hickman, C. Kos, G. Kaur, A. Sen Gupta, Platelet microparticle-inspired clot-responsive nanomedicine for targeted fibrinolysis, Biomaterials. 128 (2017) 94-108. doi:10.1016/j.biomaterials.2017.03.012.

[62] J. Xu, Y. Zhang, J. Xu, G. Liu, C. Di, X. Zhao, X. Li, Y. Li, N. Pang, C. Yang, Y. Li, B. Li, Z. Lu, M. Wang, K. Dai, R. Yan, S. Li, G. Nie, Engineered Nanoplatelets for Targeted Delivery of Plasminogen Activators to Reverse Thrombus in Multiple Mouse Thrombosis Models, Adv. Mater. 1905145 (2019) 1-14. doi:10.1002/adma.201905145.

[63] J. Hu, S. Huang, L. Zhu, W. Huang, Y. Zhao, K. Jin, Q. Zhuge, Tissue Plasminogen Activator-Porous Magnetic Microrods for Targeted Thrombolytic Therapy after Ischemic Stroke, ACS Appl. Mater. Interfaces. 10 (2018) 32988-32997. doi:10.1021/acsami.8b09423.

[64] L. Huang, J. Wang, S. Huang, F. Siaw-Debrah, M. Nyanzu, Q. Zhuge, Polyacrylic acidcoated nanoparticles loaded with recombinant tissue plasminogen activator for the treatment of mice with ischemic stroke, Biochem. Biophys. Res. Commun. 516 (2019) 565-570. doi:10.1016/j.bbrc.2019.06.079.

[65] T. Mei, A. Kim, L.B. Vong, A. Marushima, S. Puentes, Y. Matsumaru, A. Matsumura, Y. Nagasaki, Encapsulation of tissue plasminogen activator in $\mathrm{pH}$-sensitive selfassembled antioxidant nanoparticles for ischemic stroke treatment-Synergistic effect of thrombolysis and antioxidant, Biomaterials. 215 (2019) 1-12. doi:10.1016/j.biomaterials.2019.05.020.

[66] H. Kim, J.H. Ahn, M. Song, D.W. Kim, T.K. Lee, J.C. Lee, Y.M. Kim, J.D. Kim, J.H. Cho, I.K. Hwang, B.C. Yan, M.H. Won, J.H. Park, Pretreated fucoidan confers neuroprotection against transient global cerebral ischemic injury in the gerbil hippocampal CA1 area via reducing of glial cell activation and oxidative stress, Biomed. Pharmacother. 109 (2019) 1718-1727. doi:10.1016/j.biopha.2018.11.015.

[67] D. Vivien, M. Gauberti, A. Montagne, G. Defer, E. Touzé, Impact of tissue plasminogen activator on the neurovascular unit: From clinical data to experimental evidence, J. Cereb. Blood Flow Metab. 31 (2011) 2119-2134. doi:10.1038/jcbfm.2011.127.

[68] J.P.A. Ioannidis, B.Y.S. Kim, A. Trounson, How to design preclinical studies in nanomedicine and cell therapy to maximize the prospects of clinical translation, Nat. Biomed. Eng. 2 (2018) 797-809. doi:10.1038/s41551-018-0314-y.

[69] S. Yokoyama, H. Ikeda, N. Haramaki, H. Yasukawa, T. Murohara, T. Imaizumi, Platelet 
P-selectin plays an important role in arterial thrombogenesis by forming large stable platelet-leukocyte aggregates, J. Am. Coll. Cardiol. 45 (2005) 1280-1286. doi:10.1016/j.jacc.2004.12.071.

[70] S.R. Barthel, J.D. Gavino, L. Descheny, C.J. Dimitroff, Targeting selectins and selectin ligands in inflammation and cancer, Expert Opin. Ther. Targets. 11 (2007) 1473-1491. doi:10.1517/14728222.11.11.1473.

[71] Y. Shamay, M. Elkabets, H. Li, J. Shah, S. Brook, F. Wang, K. Adler, E. Baut, M. Scaltriti, P. V Jena, E.E. Gardner, J.T. Poirier, C.M. Rudin, J. Baselga, A. HaimovitzFriedman, D.A. Heller, P-selectin is a nanotherapeutic delivery target in the tumor microenvironment, Sci. Transl. Med. 8 (2016) 345ra87. doi:10.1126/scitranslmed.aaf7374.

[72] J.M. Lee, Z.-U. Shin, G.T. Mavlonov, I.Y. Abdurakhmonov, T.-H. Yi, Solid-Phase Colorimetric Method for the Quantification of Fucoidan, Appl. Biochem. Biotechnol. 168 (2012) 1019-1024. doi:10.1007/s12010-012-9837-y.

[73] C. Orset, R. Macrez, A.R. Young, D. Panthou, E. Angles-cano, E. Maubert, V. Agin, D. Vivien, Mouse Model of In Situ Thromboembolic Stroke and Reperfusion, Stroke. 38 (2007) 2771-2778. doi:10.1161/STROKEAHA.107.487520. 


\section{Supplementary Data}

Fucoidan-Functionalized Polysaccharide Submicroparticles Loaded with Alteplase for Efficient Targeted Thrombolytic Therapy

Alina Zenych ${ }^{1}$, Charlène Jacqmarcq ${ }^{2, \#}$, Rachida Aid $^{1,3, \#}$, Louise Fournier ${ }^{1}$, Laura M. Forero Ramirez $^{1}$, Thomas Bonnard ${ }^{2}$, Denis Vivien ${ }^{2,4, \#}$, Didier Letourneur ${ }^{1, \#}$, and Cédric Chauvierre ${ }^{1, *}$

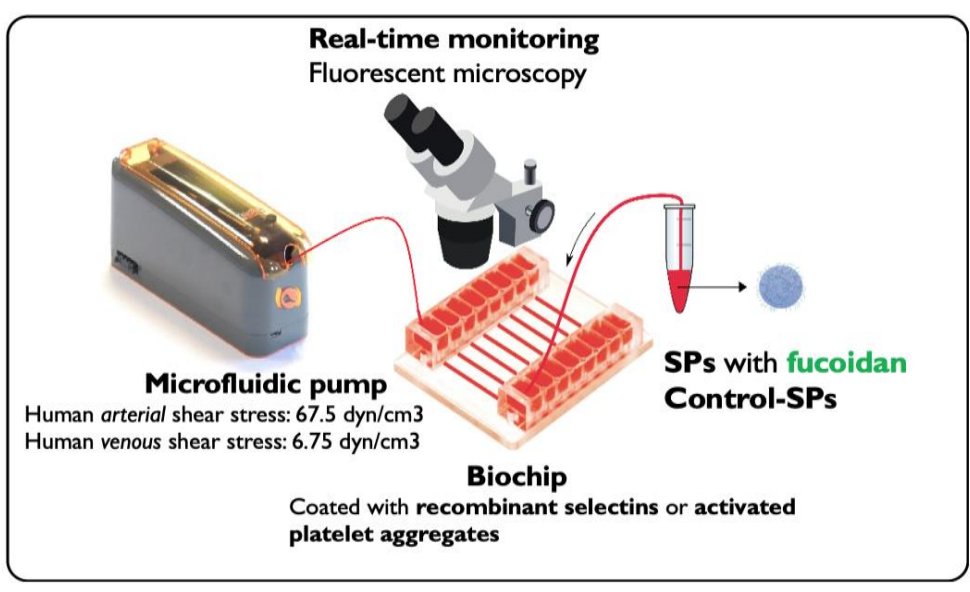

Figure S1. Schematic illustration of the in vitro targeting assay.

\section{A}
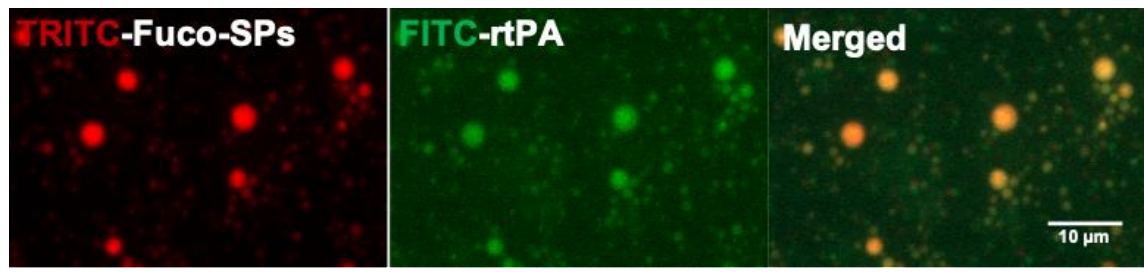

B

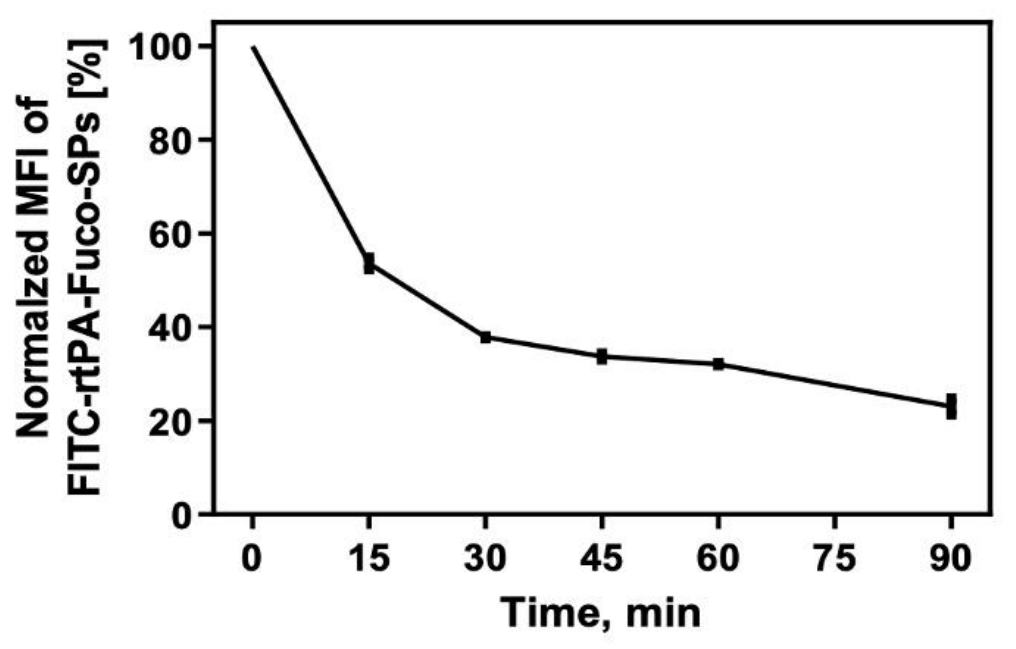


Figure S2. Loading and release of rtPA from Fuco-SPs. A. FITC-rtPA loading onto the TRITC-Fuco-SPs visualized by confocal microscopy. B. In vitro rtPA release from rtPAencapsulated Fuco-SPs by flow cytometry.

A

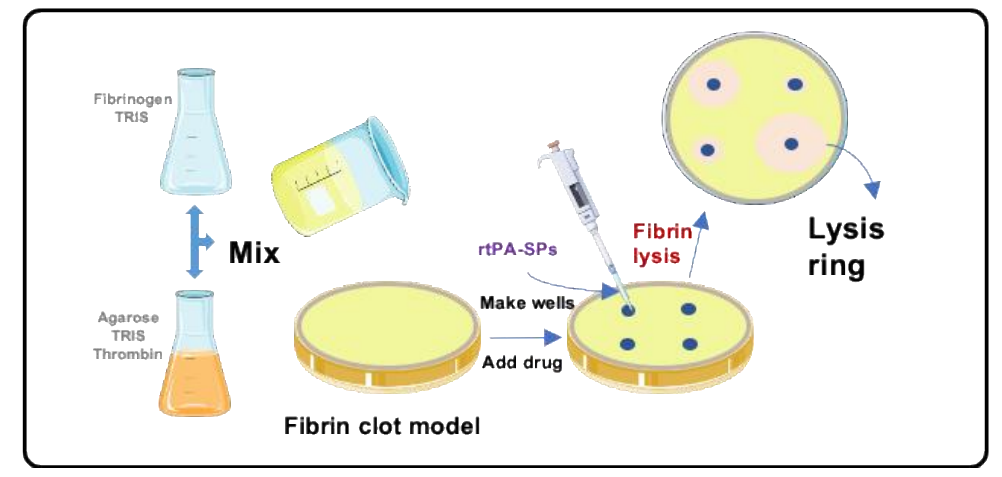

B

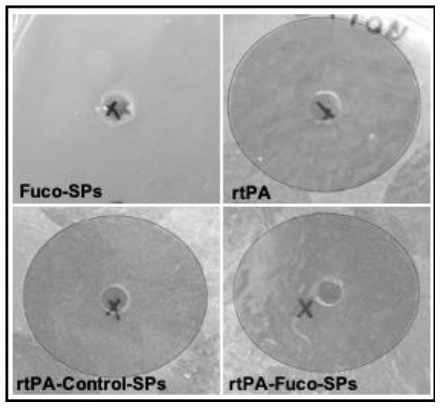

Figure S3. Fibrinolytic activity of the rtPA-loaded SPs in vitro. A. Schematic illustration of the in vitro fibrinolytic test. B. Lysed circles as the fibrinolytic potential of the SPs in vitro by a fibrin-plate agarose assay at the equal concentration of the rtPA.

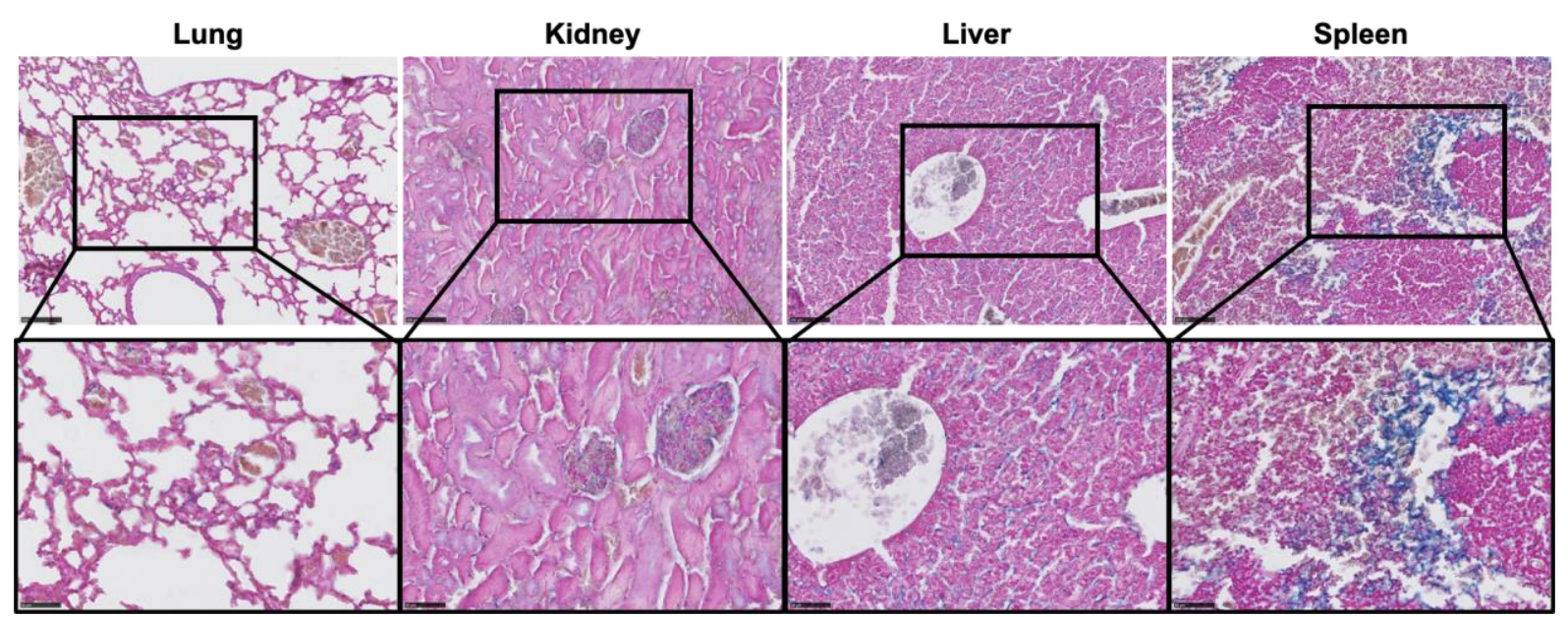

Figure S4. Histological analysis of Fuco-SPs in four organs of excretion. The cytoplasm appears pale pink, nuclei are red, and the Fuco-SPs are stained blue. Few particles were detected in the lungs, kidneys, and liver, whereas they were mainly accumulated in the spleen. The scale bar in the upper row $=100 \mu \mathrm{m}$, in the lower row (magnified image) $=50 \mu \mathrm{m}$.

A

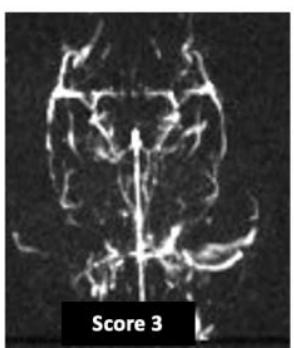

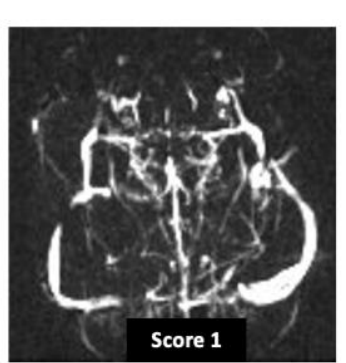

B

rtPA-Fuco-SPs

rtPA

Saline

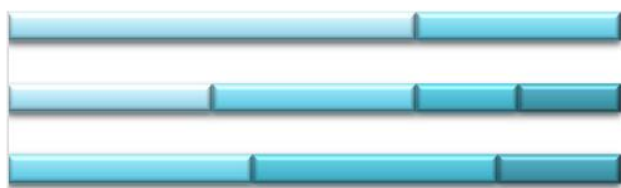

0

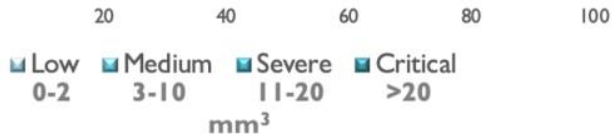


Figure S5. Therapeutic efficacy of the rtPA-Fuco-SPs in vivo. A. Merged image of the MRA scores (Score 3: full recanalization, score 1: low perfusion). B. Distribution of the cases by the infarct zone sizes by MRI $24 \mathrm{~h}$ post-stroke.

Video S1. Cerebral blood flow reperfusion monitored by the laser speckle contrast imaging during the 40-min treatment regimen with saline (A), rtPA (B), and rtPA-Fuco-SPs (C) in the ROI of ipsilateral (2) and contralateral (1) hemispheres.

Table S1. The synthesis parameters for the polysaccharide SPs.

\begin{tabular}{l|l|l|l|l|l|l|l}
\hline $\begin{array}{l}\text { Synthesis } \\
\text { method }\end{array}$ & $\begin{array}{l}\text { Polysaccharide } \\
{[\mathrm{c}], \mathrm{mg} / \mathrm{ml}}\end{array}$ & $\begin{array}{l}\text { STMP, } \\
{[\mathrm{c}],} \\
\mathrm{mg} / \mathrm{ml}\end{array}$ & $\begin{array}{l}\mathrm{NaOH} \\
{[\mathrm{c}], \mathrm{M}}\end{array}$ & $\begin{array}{l}\text { Phase } \\
\text { ratio, } \\
(\mathrm{Aq} / \\
\mathrm{Org} /)\end{array}$ & Surfactant & Homogenization & Crosslinking \\
\hline $\begin{array}{l}\text { W/O } \\
\text { emulsion / } \\
\text { crosslinking }\end{array}$ & 300 & 56.11 & 1.15 & $\begin{array}{l}4 \% \\
\mathrm{w} / \mathrm{v}\end{array}$ & $\begin{array}{l}\text { PGPR, } \\
6 \%\end{array}$ & $\begin{array}{l}30,000 \mathrm{rpm}, 4 \\
\mathrm{~min}, 4{ }^{\circ} \mathrm{C}\end{array}$ & $\begin{array}{l}{ }^{\circ} \mathrm{C} \\
\end{array}$ \\
\hline
\end{tabular}

Abbreviation: W/O, water-in-oil; Aq, aqueous; Org organic. 
Click here to access/download Movie/Animation Movie S1A - Saline.avi 
Click here to access/download Movie/Animation Movie S1B - rtPA .avi 
Click here to access/download Movie/Animation Movie S1C - rtPA-Fuco SPs.avi 